Article

\title{
An Improved Model-Based Self-Adaptive Filter for Online State-of-Charge Estimation of Li-Ion Batteries
}

\author{
Chi Zhang ${ }^{1,2}$, Fuwu Yan ${ }^{1,2}$, Changqing Du ${ }^{1,2, *}$ and Giorgio Rizzoni ${ }^{3}$ (1) \\ 1 Hubei Key Laboratory of Advanced Technology for Automotive Components, Wuhan 430070, China; \\ Zhangchi_whut@163.com (C.Z.); Yanfuwu@vip.sina.com (F.Y.) \\ 2 Hubei Collaborative Innovation Center for Automotive Components Technology, Wuhan 430070, China \\ 3 Department of Mechanical and Aerospace Engineering, The Ohio State University, 201 W 19th Ave., \\ Columbus, OH 43210, USA; rizzoni.1@osu.edu \\ * Correspondence: cq_du@whut.edu.cn; Tel.: +86-027-87850553
}

Received: 28 September 2018; Accepted: 23 October 2018; Published: 28 October 2018

\begin{abstract}
Accurate battery modeling is essential for the state-of-charge (SOC) estimation of electric vehicles, especially when vehicles are operated in dynamic processes. Temperature is a significant factor for battery characteristics, especially for the hysteresis phenomenon. Lack of existing literatures on the consideration of temperature influence in hysteresis voltage can result in errors in SOC estimation. Therefore, this study gives an insight to the equivalent circuit modeling, considering the hysteresis and temperature effects. A modified one-state hysteresis equivalent circuit model was proposed for battery modeling. The characterization of hysteresis voltage versus SOC at various temperatures was acquired by experimental tests to form a static look-up table. In addition, a strong tracking filter (STF) was applied for SOC estimation. Numerical simulations and experimental tests were performed in commercial 18650 type $\mathrm{Li}\left(\mathrm{Ni}_{1 / 3} \mathrm{Co}_{1 / 3} \mathrm{Mn}_{1 / 3}\right) \mathrm{O}_{2}$ battery. The results were systematically compared with extended Kalman filter (EKF) and unscented Kalman filter (UKF). The results of comparison showed the following: (1) the modified model has more voltage tracking capability than the original model; and (2) the modified model with STF algorithm has better accuracy, robustness against initial SOC error, voltage measurement drift, and convergence behavior than EKF and UKF.
\end{abstract}

Keywords: state of charge; strong track filter; modified one-state hysteresis model; $\mathrm{Li}\left(\mathrm{Ni}_{1 / 3} \mathrm{Co}_{1 / 3} \mathrm{Mn}_{1 / 3}\right) \mathrm{O}_{2}$ battery

\section{Introduction}

The concerns in energy crisis and global warming have driven the development of alternative energy vehicles rapidly. The Electric Vehicles (EVs), which are among the ultimate solutions for sustainable transportation, have attracted attention in aspects such as rechargeable power batteries and Battery Management System (BMS).

A key estimative parameter, state of charge (SOC) of a battery, indicates its residual capacity and reflects the remaining range of an electric vehicle. An accurate SOC estimation can not only predict the remaining range for the EVs to relieve the "range anxiety" for the drivers, but it can also help to determine an effective management strategy to avoid cell damage from over-charging and over-discharging. However, due to the complexity of the chemical and physical processes involved, characteristics of batteries present a distinct nonlinear feature, which makes their online monitoring a challenging task. Therefore, special algorithms for SOC online estimation are required. 
Previous papers coining the term 'SOC' can date back to the 1960s [1]. After several decades' efforts, a great variety of approaches have been engaged in targeting monitoring the SOC for EVs. Generally, the SOC estimation algorithms can be divided into three categories.

The first methods are based on the direct measurement, including the residual capacity method, the open-circuit voltage (OCV) method, and the Ampere-hour counting based method [2]. The residual capacity method calculates the SOC by discharging the battery to the lower cut-off voltage in controlled test equipment. It is also the most reliable method under laboratory conditions, but this is obviously not the case in online monitoring for BMS. The OCV-based method requires a long rest period, thereby not practical for EV applications. Moreover, the performance of the OCV-based method becomes severe for which the battery characteristic of voltage platform is flat, such as $\mathrm{LiFePO}_{4}$ (LFP). To the authors' knowledge, only the Ampere-hour counting based method is suitable for online monitoring. The Ampere-hour counting method estimates the SOC by integrating the flow-in and flow-out current of the battery. This method reportedly has several theoretical limitations and is an open loop method that cannot correct the accumulative error caused by current measuring transducers drift. In addition, the estimation accuracy is dependent on the initial SOC. This method has low robustness against the acquired signal quality, as well as initial SOC information.

The second types are the machine learning methods, such as the Artificial Neural Network (ANN), Fuzzy Logic (FL), and Support Vector Machine (SVM). These methods are also called "black box" model by which these do not need the detailed information of the battery system. Mahmoud Ismail et al. [3,4] developed an ANN SOC estimator for commercial Li-ion battery. The network is trained by the input of current and voltage, and the output is the battery SOC. The algorithm is validated by the benchmark driving cycles and can achieve a relatively high degree of accuracy. Claudio Burgos et al. [5] introduced a fuzzy-based model to characterize the discharge behavior of lead-acid batteries. Du Jiani et al. [6] presented a methodology of FL to describe the equivalent circuit model parameters on SOC and temperature effects. The SVM model is also a smart choice for SOC estimation, which has been used in several literatures [7-11]. Although much research focused on the machine learning methods, some of which have shown good performance, the common shortfall of these methods is the heavy computational burden that makes the online implementation unpractical.

The last algorithms are based on the control-oriented battery model. The model-based estimators are used to calculate the SOC by characterizing the battery behavior through measurable signals like current, terminal voltage, and temperature. These model-based estimators include the electrochemical model, the Equivalent Circuit Model (ECM), and the empirical model. The electrochemical model is the most precise model among the others, wherein it describes the electrochemical reaction processes of the battery by adopting a set of partial differential equations. However, it is also the most complicated model for the limited BMS computational resource. Meanwhile, the empirical model usually has a simple model structure with a low computation demand. However, a large number of experiments are required to build a database. Moreover, for most BMS applications, the ECM is used as a solution, because it meets the best compromise between the accuracy and model complexity.

Currently, the combinations of ECM and system filtering theory have drawn continuous attention by scholars and industry developers. Among all the system filtering theories, the Kalman filter $(\mathrm{KF})$ is the most frequently used [12]. Compared with other system filtering algorithms, the KF method does not need an accurate initial value of SOC because the result will gradually approach the optimal value and the current measurement error will be updated during the operational process of the algorithms. Meanwhile, it is a closed-loop observer, and it can achieve accurate and continuous estimation performance during the whole range of battery operations. The abovementioned advantages make KF a promising solution for BMS application implementation. Moreover, the ordinary KF is only suitable for linear systems, whereas the BMS applications require the use of more complex and nonlinear algorithms. Several advanced modifications have been proposed, such as an extended Kalman filter (EKF), unscented Kalman filter (UKF), and Central-Difference Kalman filter (CDKF). Gregory L. Plett was the first to establish EKF for SOC estimation [13-16]. Based on his pioneering 
work, a great variety of research concerning the applications of EKF to the non-linear system have been reported in several literatures [17-22]. However, EKF is essentially a first-order Taylor series expansion of the state-space equations that have the theoretical limitation of estimation accuracy under the case of high dynamic current change. In addition, the EKF needs to calculate the Jacobi matrix, which leads to the algorithm being inefficient or even numerical instabilities in the implementation of a low-cost microcontroller. As an alternative system linear approach, the UKF was developed to improve the estimation accuracy and to compute reliability Theoretically, all KF variants require the knowledge of process and measurement noise covariance. Inappropriate tuning parameters may lead to low convergence and high oscillation. Therefore, adaptive technology has been introduced to combine with the KFs. Sun et al. [23] presented an adaptive UKF to estimate the SOC of EV applications. The adaptive adjustment of the noise covariance was dealt by covariance matching methods. Xiong et al. [24] proposed a data-driven-based approach for SOC estimation by employing an adaptive EKF algorithm. Their methods achieved good accuracy and convergence for different types of lithium-ion batteries.

To summarize, a modern smart algorithm for online SOC estimation in a BMS application requires the following characteristics: capable of describing the first- or second-order nonlinear behavior of battery system under dynamic excitation; adaptive adjustment for system noise matrices and high converge robustness against the drastic change of current; numerical stability; and ease of implementation in an embedded chip.

To address the abovementioned problems, an optimized model-based algorithm combined with Strong Tracking Filter (STF) was put forward for online SOC monitoring. The one-state hysteresis model was applied to state-space function and the hysteresis voltage was considered in terms of temperature. The proposed algorithm has the following advantages: (1) strong robustness against model uncertainties; (2) strong tracking ability of the mutation status; and (3) a moderate computational burden. Various experimental tests were designed to validate the proposed approach. The comparisons among the EKF, UKF, and STF were carried out to evaluate the performance of the proposed algorithm.

The remainder of this paper was organized as follows: The battery modeling was introduced in Section 2. In Section 3, the experimental setup and identification results were demonstrated in detail. In Section 4, EKF, UKF, and STF were proposed for the implementation of SOC estimation. Section 5 illustrated the experimental results in comparison with EKF and UKF in the aspects of estimation accuracy, robustness, and convergence behavior. Section 6 presents the conclusions.

\section{Battery Modeling}

For control-oriented battery online monitoring, a precise battery state-space model must be available. As mentioned, the ECM is the most widely used choice in combination with the KFs. The ECM is based on the Thevenin's theorem, which approximates the battery's electrical behavior through a voltage source and some resistances and capacitors. The accuracy of ECM was enhanced by adding extra resistance-capacitance terms (RC network) into the circuits. However, the complication of the model structure could lead to inefficient parameters' identification and low real-time computation. Obviously, a reasonable model must consider simulation accuracy, parameterization efficiency, and computation burden. Additionally, hysteresis is also a very significant variable for lithium-ion batteries [25-28]. However, studies for this variable are lacking as it is rarely being considered. In this paper, the hysteresis model was used. As shown in Figure 1, the proposed model consists of an Electro-Motive Force (EMF) voltage resource, a hysteresis voltage resource, a resistor $R_{0}$, and a $\mathrm{RC}$ network connected in series. The EMF voltage resource and the hysteresis voltage resource are together to form a controlled voltage source $-U_{\mathrm{oc}}(\mathrm{OCV}) . R_{0}$ is the ohmic resistance. It represents the instantaneous voltage variation caused by the electrolyte and the active mass. $R_{1}$ and $C_{1}$ represent the polarization resistance and polarization capacitance, respectively. These are also used to depict the transient response of the cell caused by double-layer capacity effects. 


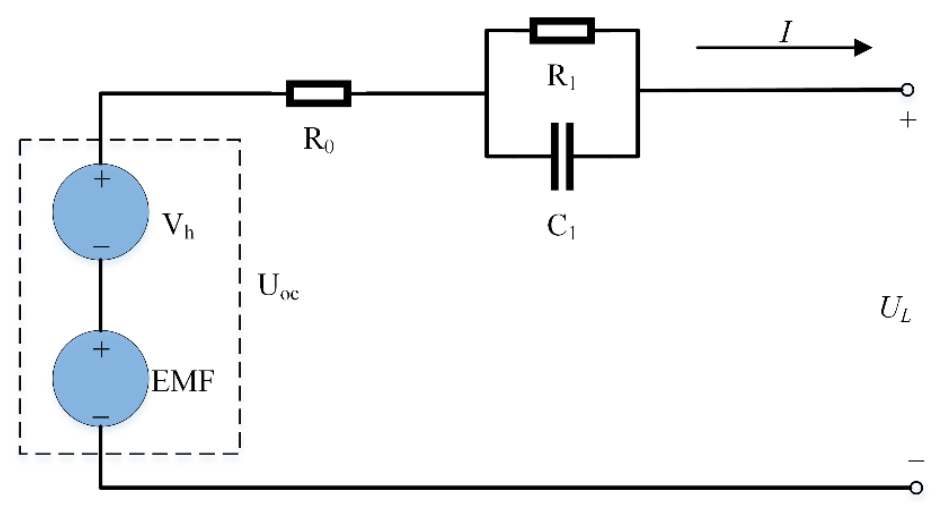

Figure 1. The schematic diagram of the one-state hysteresis equivalent circuit model (ECM).

The hysteresis effect between the charge and discharge boundary curves is illustrated in Figure 2a. The curves are obtained by charging/discharging the cell at C/25 rate (a current corresponding to the manufacturer's rated capacity for a $25 \mathrm{~h}$ discharge to low cut-off voltage) at a temperature of $30{ }^{\circ} \mathrm{C} ; 30^{\circ} \mathrm{C}$ is the standard temperature according to the latest U. S. Advanced Battery Consortium (USABC) battery test manual [29]. The charge voltage is the upper curve and the discharge voltage is the lower curve. At such low rate, we believed that the voltage drop was caused by ohmic resistance, and polarization is small enough to ignore (less than $1 \mathrm{mV}$ in this case). Thus, the voltage difference at each SOC is caused by the hysteresis phenomenon.

The space function equations corresponding to Figure 1 are derived as follows:

First, according to the Kirchhoff voltage law, the governing equation of the cell model can be derived as follows:

$$
\left\{\begin{array}{l}
U_{L}=U_{o c}(S O C, T)+I R_{0}+U_{1}+h \\
\overline{U_{1}}=-\frac{1}{R_{1} C_{1}} U_{1}+\frac{1}{C_{1}} I
\end{array}\right.
$$

where $U_{L}$ is the terminal voltage of the cell. EMF is just a theoretical concept, where the EMF is approximated by $U_{o c}(S O C, T)$ after an hour rest, which is the function of SOC and temperature. $U_{1}$ is the voltage over the RC network and $h$ is the voltage caused by hysteresis. I is the main circuit current. The charging process is positive, and the discharging process is negative. $R_{1}$ is the polarization resistance and $C_{1}$ is the polarization capacitance. $h$ is the hysteresis voltage, it is the function of the SOC and temperature.

The SOC of the cell can be expressed as follows:

$$
\operatorname{SOC}(\mathrm{k})=\operatorname{SOC}(0)+\frac{\int_{0}^{k} \eta I d t}{\mathrm{C}_{\mathrm{N}}}
$$

where $\mathrm{SOC}(0)$ is the initial SOC value of the cell; $\mathrm{SOC}(k)$ is the current SOC value at time $k ; \mathrm{C}_{\mathrm{N}}$ is the nominal capacity and the function of the discharge rate and temperature; $\eta$ is the coulomb efficiency, the function of current and temperature [30].

To implement the one-state hysteresis model to KFs, the above equations need to be discretized to the following linear discrete form [31]:

$$
\begin{gathered}
U_{L, k}=U_{o c, k}+R_{0} I_{k}+U_{1, k}+h_{k} \\
U_{1, k}=\exp \left(-\frac{T_{S}}{R_{1} C_{1}}\right) U_{1, k-1}+\left[1-\exp \left(-\frac{T_{S}}{R_{1} C_{1}}\right)\right] R_{1} I_{k-1} \\
S O C_{k}=S O C_{k-1}+\frac{\eta T_{s}}{C_{N}} I_{k-1}
\end{gathered}
$$



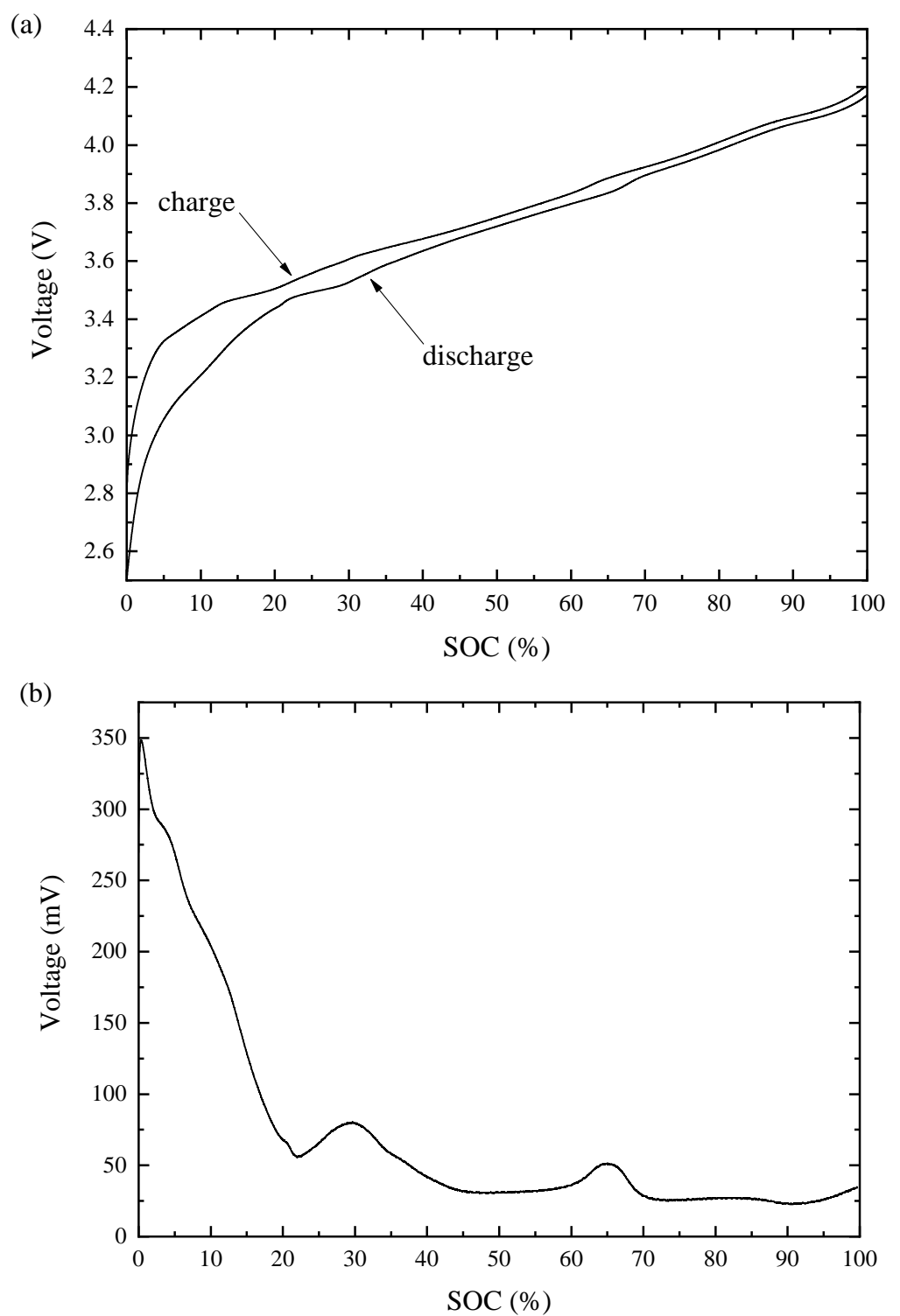

Figure 2. Open circuit voltage $(\mathrm{OCV})$ discharge and charge boundary at $30{ }^{\circ} \mathrm{C}$ : (a) the discharge and charge curve; (b) the hysteresis level.

For the hysteresis state function, let $h(S, t)$ be the hysteresis voltage as the function of SOC and time, and $\hat{S}=d S / d t$.

$$
\frac{d h(S, t)}{d S}=\gamma \operatorname{sgn}(\hat{S})(M(S, \hat{S})-h(S, t))
$$

where $M(S, \hat{S})$ is the maximum hysteresis voltage as a function of SOC and SOC changing rate. $\gamma$ is a positive constant to tune the rate of decay, and $\operatorname{sgn}(\hat{S})$ is to make the equation stable for charge and discharge process.

Since $d h(S, t) / d t=d h(S, t) / d S \times d S / d t$, the deformation of Equation (6) can be expressed by Equation (7):

$$
\frac{d h(S, t)}{d t}=\gamma \operatorname{sgn}(\hat{S})(M(S, \hat{S})-h(S, t)) \times \frac{d S}{d t}
$$

According to Equation (2), it is easy to conclude that $d S / d t=\eta I / C_{N}$. Meanwhile, $\operatorname{sgn}(\hat{S}) \times$ $d S / d t=|d S / d t|$. Then, Equation (7) is rewritten by:

$$
\hat{h}(t)=\left|\frac{\eta I(t) \gamma}{C_{N}}\right| M(S, \hat{S})-\left|\frac{\eta I(t) \gamma}{C_{N}}\right| h(t)
$$


And then, discretize Equation (8) to:

$$
h_{k}=\exp \left(-\left|\frac{\eta I(t) \gamma}{C_{N}}\right|\right) h_{k-1}+\left(1-\exp \left(-\left|\frac{\eta I(t) \gamma}{C_{N}}\right|\right)\right) M(S, \hat{S})
$$

\section{Battery Experiments}

\subsection{Test Bench}

As shown in Figure 3, an experimental test bench was established to study the characteristics of the lithium-ion battery. The experimental setup consisted of the following: (1) a set of Sony US18650VTC5a type cells (Sony Corp., Tokyo, Japan) with $\mathrm{Li}\left(\mathrm{Ni}_{1 / 3} \mathrm{Co}_{1 / 3} \mathrm{Mn}_{1 / 3}\right) \mathrm{O}_{2}(\mathrm{NCM})$ cathode and graphite anode; (2) a thermal chamber with temperature control; (3) current and voltage sensors; (4) a battery test station; (5) a host computer with software to set up the database; and (6) a Matlab 2017b (MathWorks Inc., Natick, MA, USA) to run the model. The cell is a commercial battery that is used for high specific energy demand application with a nominal voltage of $3.7 \mathrm{~V}$ and a nominal capacity of 2.5 Ah. The main characteristics of nominal voltage and nominal capacity are shown in Table 1. For the tests, the temperature chamber was set at $-20^{\circ} \mathrm{C},-10^{\circ} \mathrm{C}, 0{ }^{\circ} \mathrm{C}, 10^{\circ} \mathrm{C}, 20^{\circ} \mathrm{C}, 30^{\circ} \mathrm{C}, 40{ }^{\circ} \mathrm{C}$, $50^{\circ} \mathrm{C}$, respectively. The battery test station (NEWARE BTS4002, Shenzhen, China) was used for test profile control. Each channel of the BTS was capable of $\pm 20 \mathrm{~A}$ current and $+5 \mathrm{~V}$ voltage. The accuracy of current and voltage measurement was $50 \mathrm{~mA}$ and $10 \mathrm{mV}$, respectively.

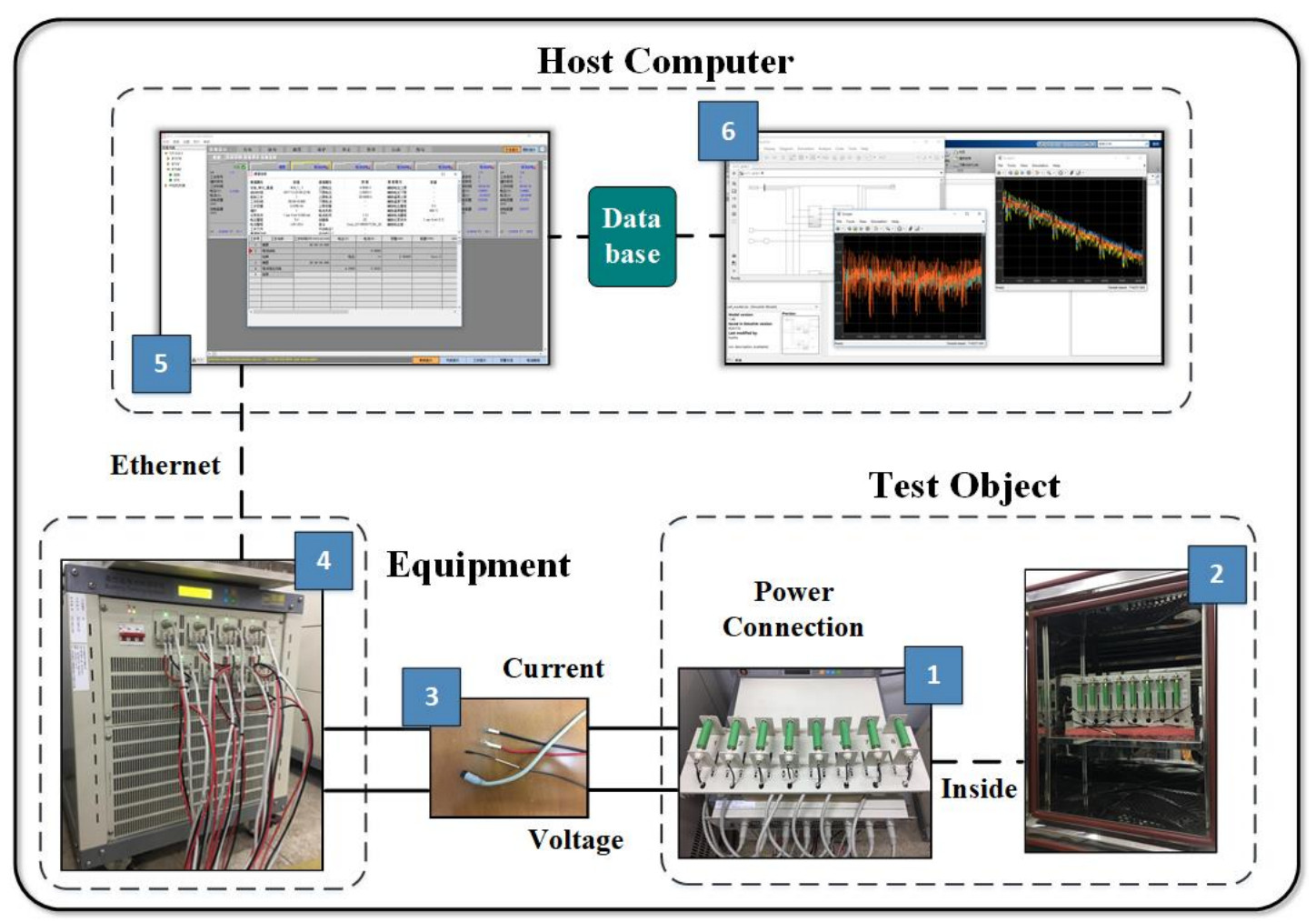

Figure 3. Schematic diagram of the battery test bench.

Table 1. Characteristics of Sony US18650VTC5a cell.

\begin{tabular}{cc}
\hline Item & Rating \\
\hline Capacity & $2.5 \mathrm{Ah}$ \\
End of discharge voltage & $2.5 \mathrm{~V}$ \\
Maximum charge voltage & $4.2 \mathrm{~V}$ \\
Maximum discharge current rate & $12 \mathrm{C}$ \\
\hline
\end{tabular}




\subsection{Test Schedule}

Overall, the tests involved in this study were categorized into one of two types, namely, a parameterization and a validation test schedule. The parameterization test includes hysteresis voltage test and a series of HPPC (Hybrid Pulse Power Characterization) tests.

The hysteresis voltage is a considerable factor for battery modeling. Thus, the hysteresis voltage test was conducted at various temperatures to improve the model accuracy. During the hysteresis voltage test, the cells were discharged by $1 / 25 \mathrm{C}$ constant current, until the low cut-off voltage $(2.5 \mathrm{~V})$ was reached. After the $1 \mathrm{~h}$ period rest, the cells were recharged by the same $1 / 25 \mathrm{C}$ rate until the upper cut-off voltage $(4.2 \mathrm{~V})$. This test was carried out at temperatures of $0{ }^{\circ} \mathrm{C}, 15^{\circ} \mathrm{C}, 30^{\circ} \mathrm{C}$, and $45^{\circ} \mathrm{C}$. There were 4 cycles involved in total, all the discharge and chaege cycles was carried out by constant current (CC) method.

The HPPC test profile as referred to [29], was composed of a series of hybrid pulse power steps with $1 \mathrm{~h}$ rest at each SOC point. The current profile and the voltage response of a HPPC microcycle are shown in Figure 4. The HPPC test was carried out with $1 \mathrm{C}$ rate under the temperatures ranging from $0{ }^{\circ} \mathrm{C}$ to $50{ }^{\circ} \mathrm{C}$, with an interval of $10^{\circ} \mathrm{C}$. In HPPC test, the model parameters of $\mathrm{OCV}, \mathrm{R}_{0}, \mathrm{R}_{1}, \mathrm{C}_{1}$ were identified online. The identification results were discussed in Section 3.4.
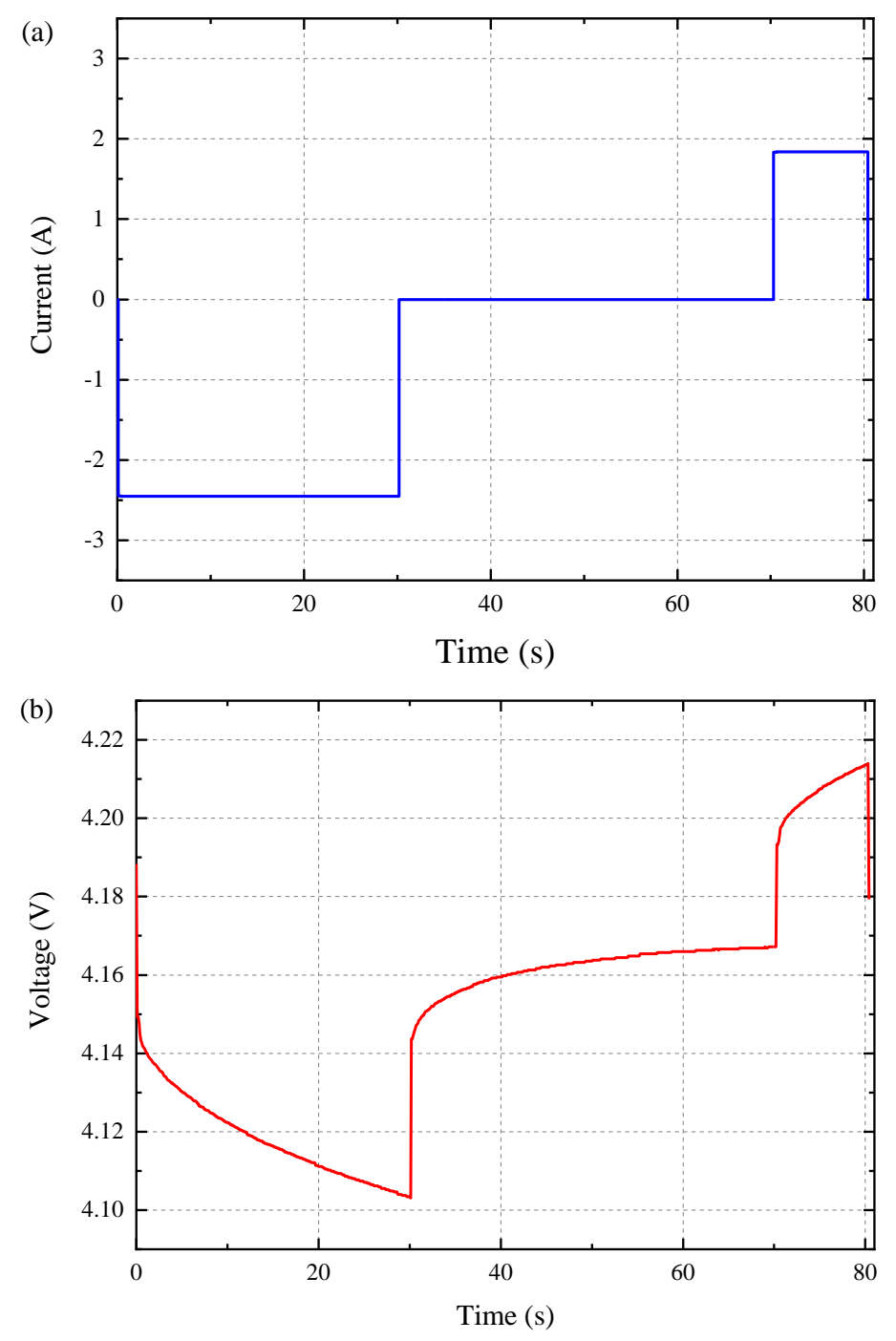

Figure 4. Current profile and voltage response of a hybrid pulse power characterization (HPPC) microcycle. 
The purpose of the validation test was to evaluate the performance of the modified model, as well as the SOC approach, which will be introduced in the next section. Therefore, a current sequence derived from the Federal Urban Driving Schedule (FUDS) cycle was used in this study. FUDS is a dynamic current load which is designed to simulate the battery operation case in EV applications. In summary, the entire profile consisted of nine typical FUDS microcycles; a 1/3 C constant current discharge between the FUDS cycles and the constant current discharge was a rest period of one hour. To implement the FUDS to the cells, the battery size factor (BSF) was scaled down to $9.25 \mathrm{Wh}$. The cells were fully charged before the test and were discharged to the low cut-off voltage of $2.5 \mathrm{~V}$ at last. Therefore, the "true value" of SOC was calculated by the accumulative Ampere-hours flowing in and out of the battery. Figure 5 shows the sampled current and voltage of the validation test profile. The validation test was run at the temperature ranges from $-20{ }^{\circ} \mathrm{C}$ to $50^{\circ} \mathrm{C}$. It is worth pointing out that the test should be terminated immediately when the low cut-off voltage of $2.5 \mathrm{~V}$ was reached.
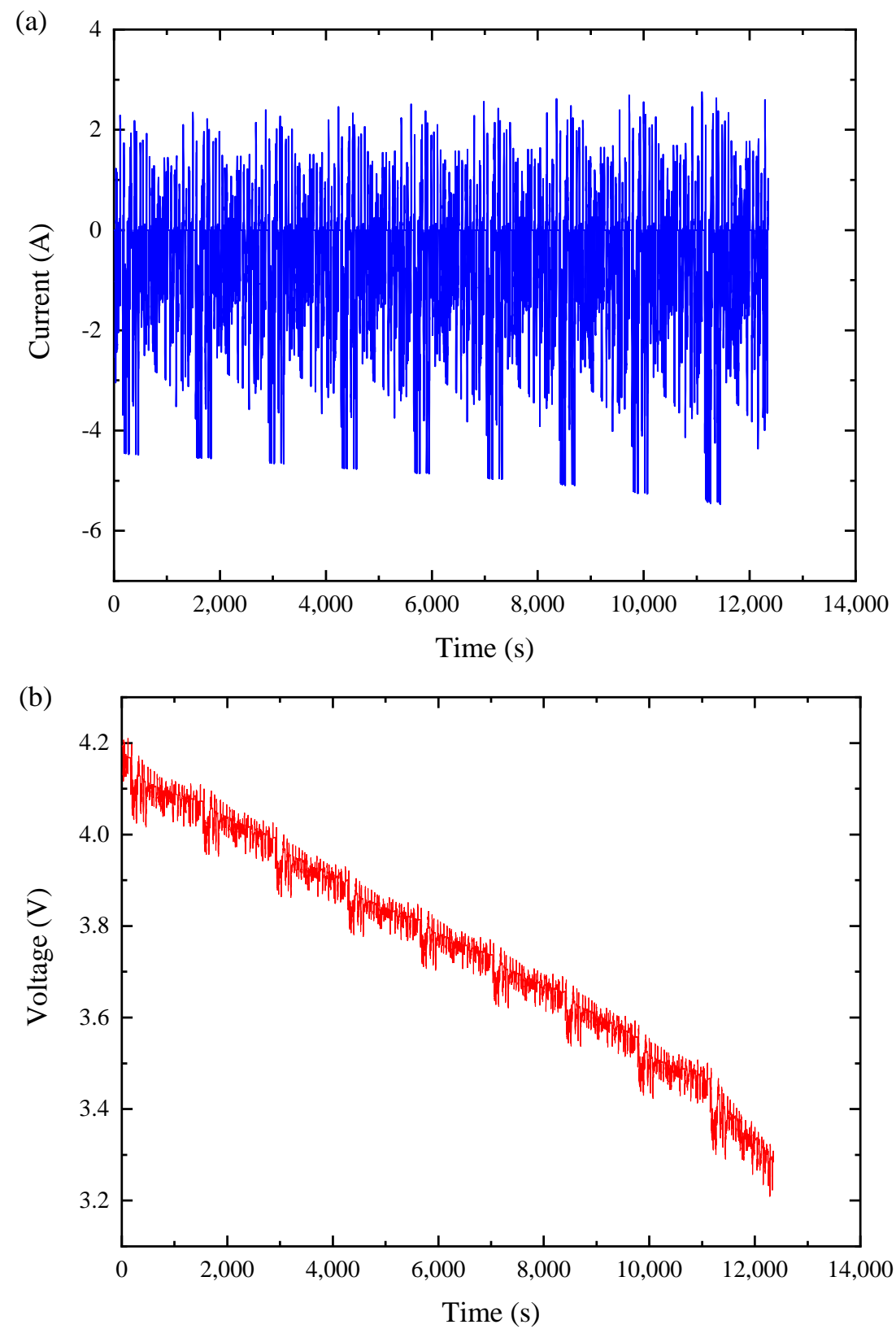

Figure 5. The profile of validation test cycles: (a) current profile; (b) voltage response. 


\subsection{Parameters Identification}

In this work, two types of battery parameters are needed to be identified, namely the function of $h_{v}=f(S O C, T)$, and the intrinsic parameters of the ECM which were identified offline and online, respectively. Let $\xi=\left[O C V, R_{0}^{+}, R_{0}^{-}, R_{1}, C_{1}\right]$ be the online identification parameters. The current and voltage response of HPPC data were selected and identified by using recursive least square method (RLS). For online identification, because OCV is the function of SOC, an OCV function is needed for curve fitting. Here, the model proposed by Plett [14] was employed. The OCV at the end of the $1 \mathrm{~h}$ rest period of HPPC at each SOC was adopted.

$$
\mathrm{OCV}=k_{0}-\frac{k_{1}}{S O C}-k_{2} S O C+k_{3} \operatorname{In}(S O C)+k_{4} \operatorname{In}(1-S O C)
$$

Taking $0{ }^{\circ} \mathrm{C}$ as an example, the measured OCV and the nonlinear fitted function are displayed in Figure 6a. In Figure 6b, the generalized residual was utilized to describe the degree of the fitting. The data at the rest of the temperatures has the similar results.
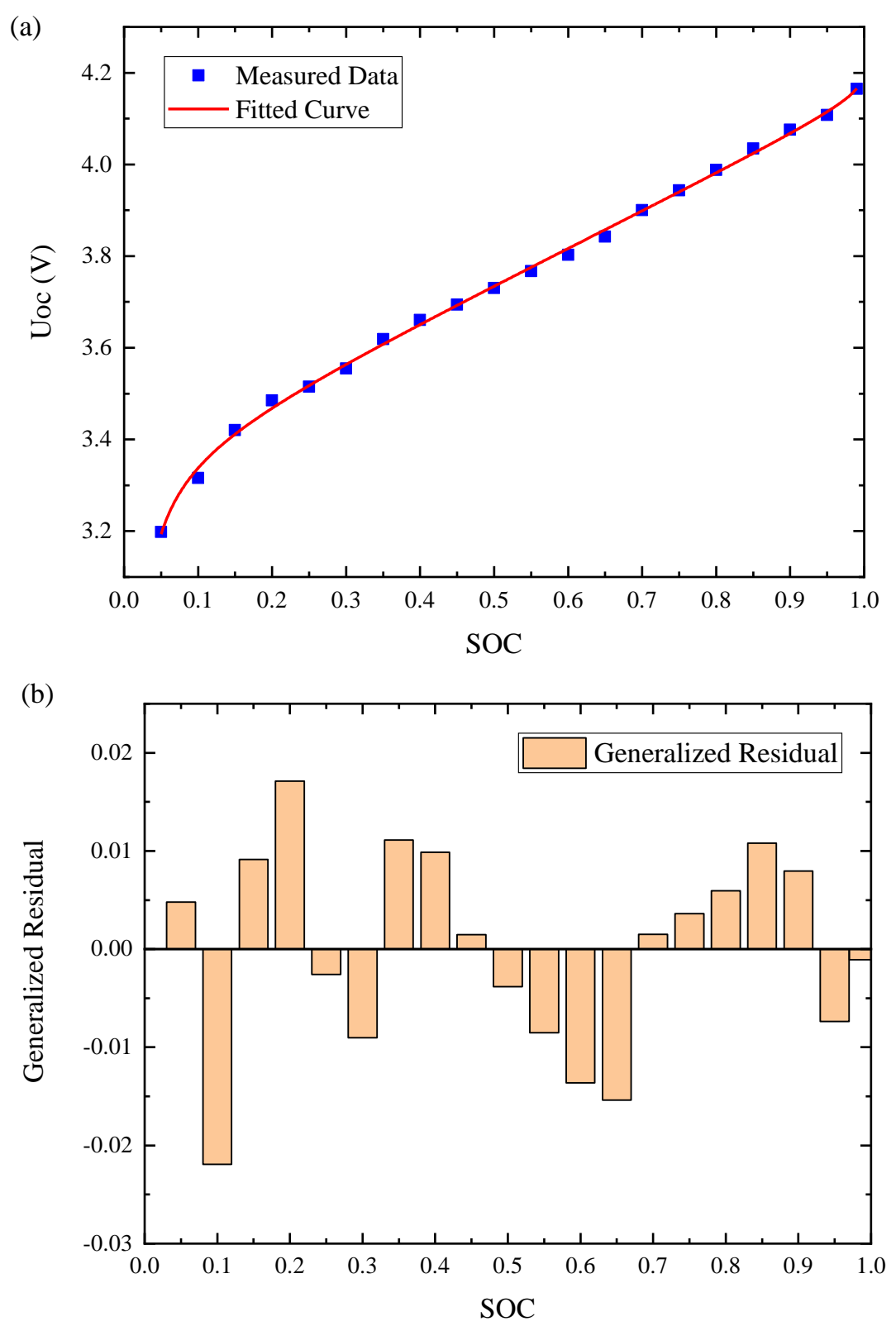

Figure 6. The OCV result at temperature of $0{ }^{\circ} \mathrm{C}$ : (a) fitting curve; (b) the generalized residual. 
To further improve the model accuracy, the OCV was corrected by $h_{v}$; the $h_{v}$ is preprocessed to form an offline $h_{v}$-SOC-T look-up table. As shown in Figure 7, the OCV boundaries of discharge and charge process and hysteresis level under various temperatures were depicted.
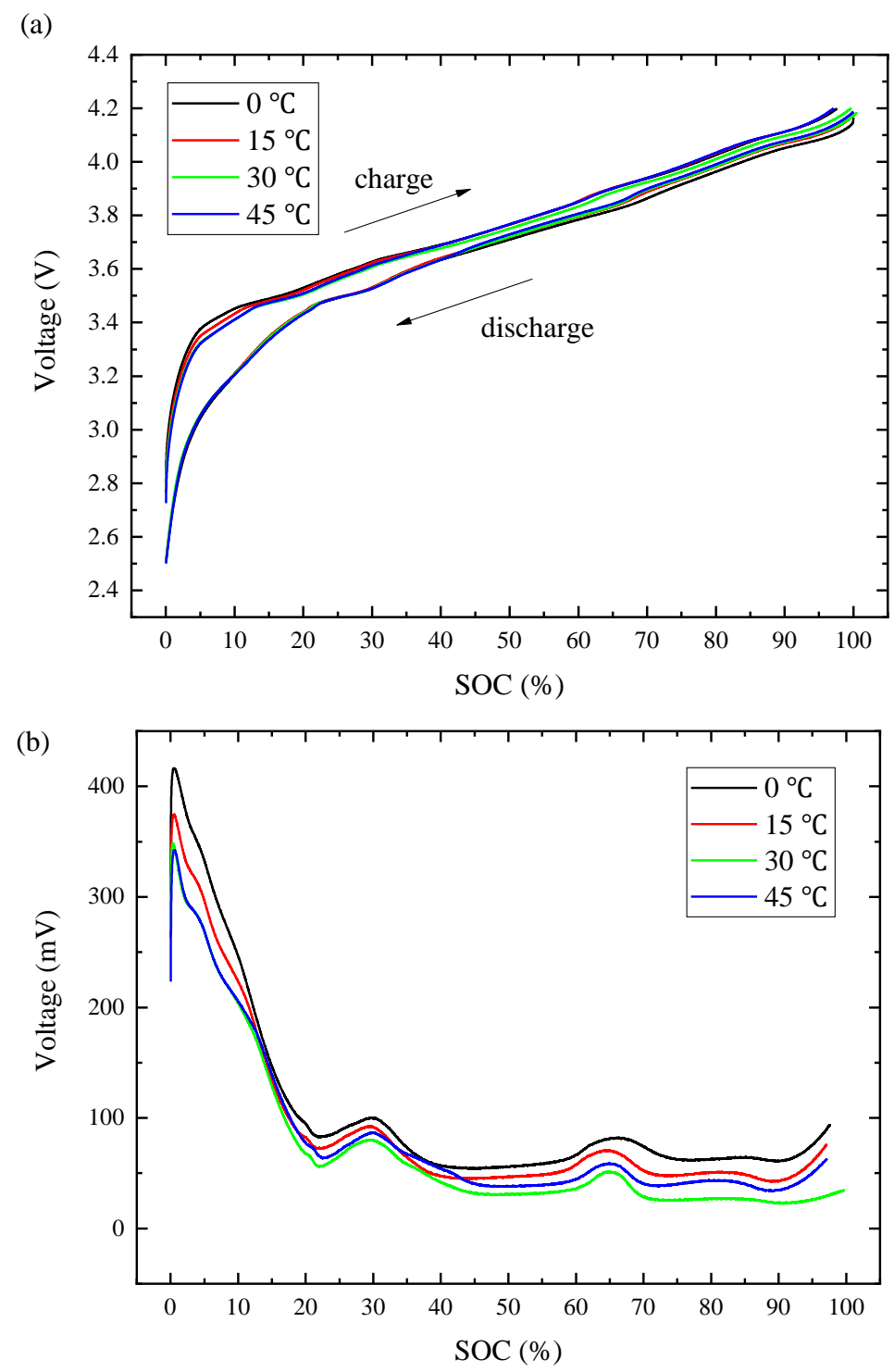

Figure 7. The OCV and hysteresis level under different temperatures: (a) the OCV boundaries of discharge and charge process at different temperatures; (b) the hysteresis voltage level.

\subsection{Identification Results}

The identified model was validated by the validation test profile. Here, we defined the original model as lacking hysteresis terms, whereas the improved model as considering hysteresis and temperature effect. The quantitative evaluation of goodness-of-fit of the model, MAE (Mean Absolute Error), and RMSE (Root Mean Squared Error) were introduced by the following equations:

$$
\begin{gathered}
\text { MAE }=\left(\frac{\int_{k=0}^{n}|\hat{S}(k)-S(k)|}{n}\right) \times 100 \% \\
\operatorname{RMSE}=\left(\sqrt{\frac{\sum_{k=0}^{n}(\hat{S}(k)-S(k))}{n}}\right) \times 100 \%
\end{gathered}
$$


where $S(k)$ is the experimental data and $\hat{S}(k)$ is the estimated value at step k.

Again, taking $0{ }^{\circ} \mathrm{C}$ for example, the simulated voltage by the original model and improved model are shown in Figure 8a and the errors of the two models are depicted in Figure 8b. For the profile at $0{ }^{\circ} \mathrm{C}$, the MAE of the original model and improved model were $47.9 \mathrm{mV}$ and $24.0 \mathrm{mV}$, respectively; and the RMSE of the original model and improved model were $59.8 \mathrm{mV}$ and $29.9 \mathrm{mV}$, respectively. The MAE and RMSE at all temperatures are illustrated in Figure 9. Generally, the MAE and RMSE of both the original model and improved model decreased as the temperatures increased. However, as the temperature moves farther away from $30^{\circ} \mathrm{C}$, the performance of the proposed model becomes better than the original model. Unlike the proposed model, the original model's parameters were identified at $0{ }^{\circ} \mathrm{C}$, thus the lack of parameters' correction in terms of the temperatures led the above results.

(a)

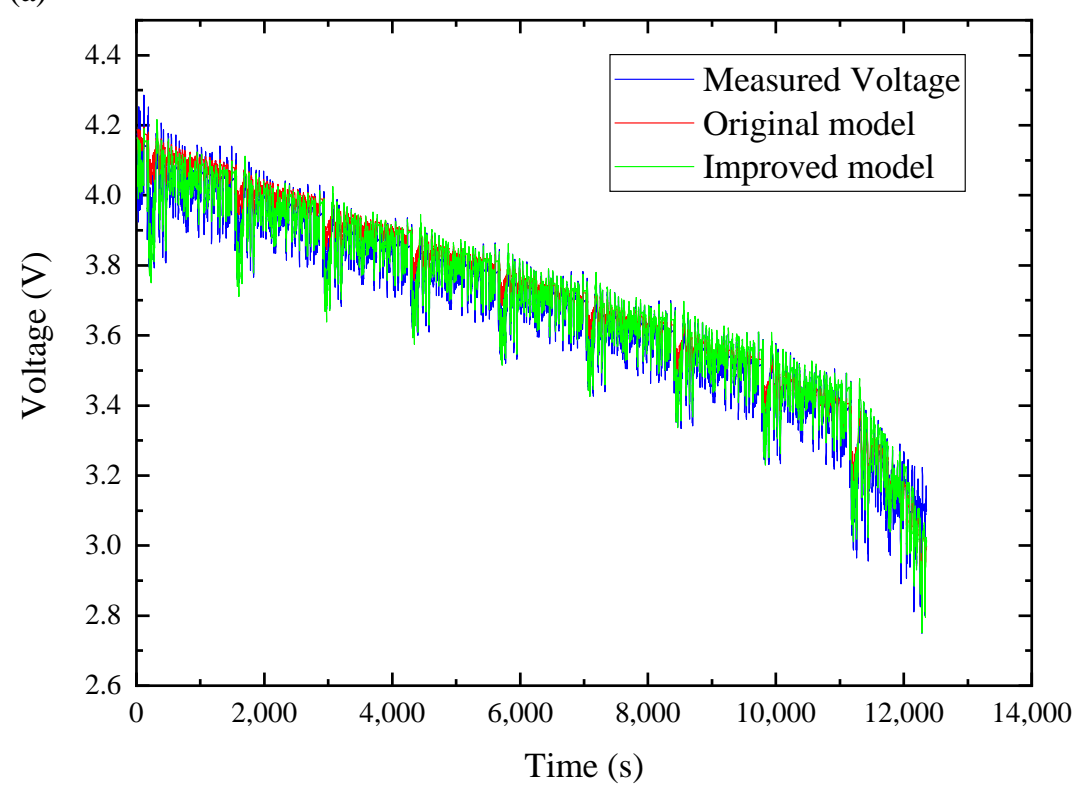

(b)

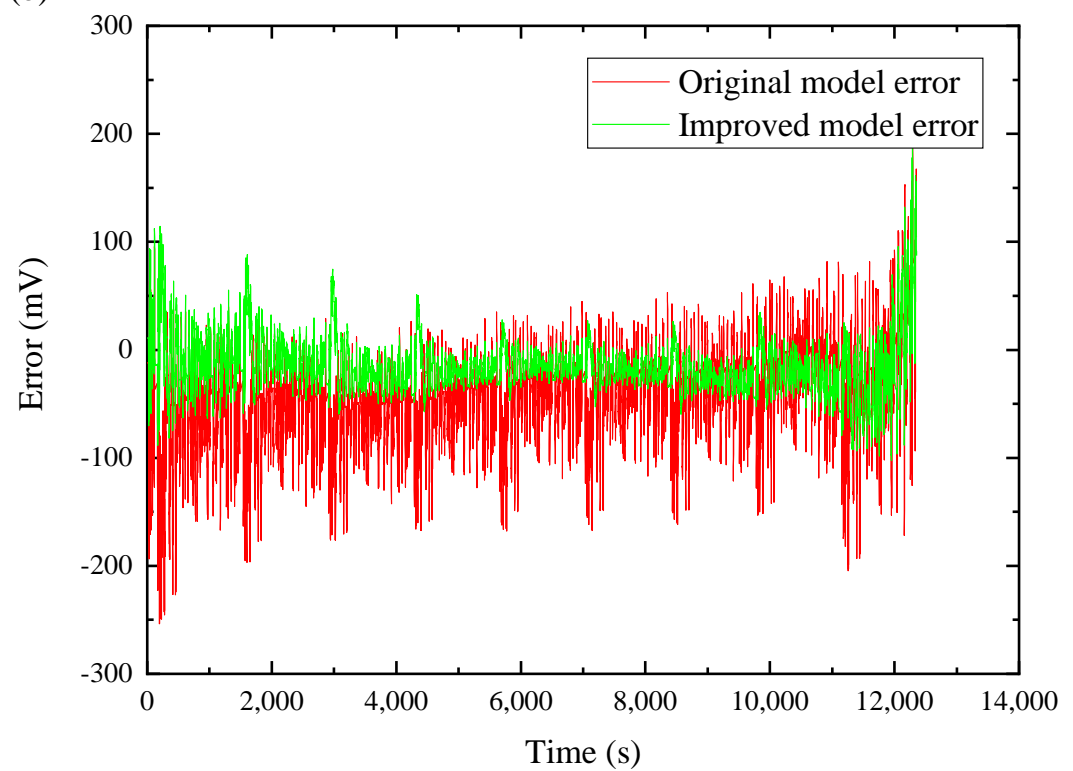

Figure 8. Comparison between measured data and model results at $0{ }^{\circ} \mathrm{C}$ : (a) voltage of experimental, original model and improved model; (b) estimated error of the original and improved model. 


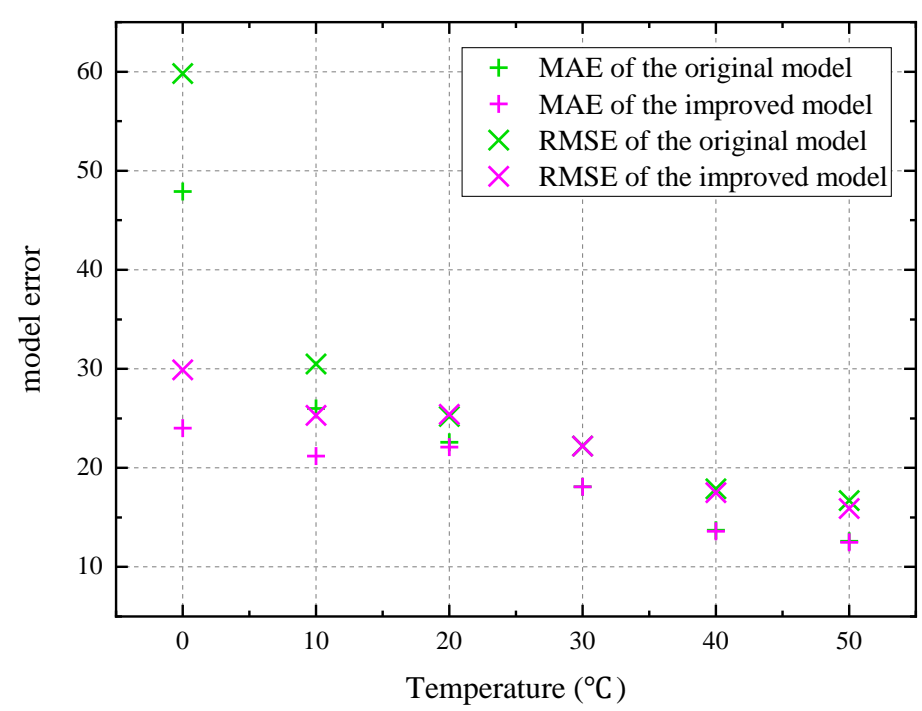

Figure 9. The mean absolute error (MAE) and root mean squared error (RMSE) of the original model and improved model.

\section{SOC Estimation Based on EKF and UKF}

According to Equations (3)-(5) and (9), the state-space equation is as follows:

$$
\begin{aligned}
& {\left[\begin{array}{c}
U_{1, k} \\
h_{k} \\
S O C_{k}
\end{array}\right]} \\
& =\left[\begin{array}{ccc}
\exp \left(-\frac{T_{s}}{R_{1} C_{1}}\right) & 0 & 0 \\
0 & \exp \left(-\left|\frac{\eta I_{k} \gamma T_{s}}{C_{N}}\right|\right) & 0 \\
0 & 0 & 1
\end{array}\right]\left[\begin{array}{c}
U_{1, k-1} \\
h_{k-1} \\
S O C_{k-1}
\end{array}\right] \\
& +\left[\begin{array}{ccc}
{\left[\begin{array}{cc}
1-\exp \left(-\frac{T_{s}}{R_{1} C_{1}}\right)
\end{array}\right] R_{1}} & 0 & 0 \\
0 & \left(1-\exp \left(-\left|\frac{\eta I(t) \gamma T_{s}}{C_{N}}\right|\right)\right) & 0 \\
0 & 0 & \frac{\eta T_{s}}{C_{N}}
\end{array}\right]\left[\begin{array}{c}
I_{k-1} \\
M(S, \hat{S}) \\
I_{k-1}
\end{array}\right]+\omega_{k-1} \\
& U_{L, k}=U_{o c, k}+R_{0} I_{k}+U_{1, k}+h_{k}+v_{k}
\end{aligned}
$$

where $\omega_{k-1}$ is the system process noise and $v_{k}$ is the measurement noise. Based on the above state-space equations, the proposed model can be implemented on EKF and UKF, respectively

\subsection{Extended Kalman Filtering}

As there have been plenty of published literatures on the EKF approach, the algorithm principle was shortly introduced, to avoid repetitions. The nonlinear state-space equation and measurement discretized equation can be represented as follows:

$$
\begin{gathered}
x_{k+1}=f\left(x_{k}, u_{k}\right)+\Gamma_{k} \omega_{k} \\
y_{k+1}=g\left(x_{k}, u_{k}\right)+v_{k}
\end{gathered}
$$

where $f\left(x_{k}, u_{k}\right)$ is the nonlinear state function and $g\left(x_{k}, u_{k}\right)$ is the nonlinear measurement function. Then, $\hat{A}_{k}=\left.\frac{\partial f\left(x_{k}, u_{k}\right)}{\partial x_{k}}\right|_{x_{k}=\hat{x}_{k}}, \hat{C}_{k}=\left.\frac{\partial g\left(x_{k}, u_{k}\right)}{\partial x_{k}}\right|_{x_{k}=\hat{x}_{k}}$ was defined as the Jacobian matrix of $f(\cdot)$ and $g(\cdot)$, respectively.

The EKF is summarized in Table 2 as referenced in [13]. 
Table 2. Summary of the extended Kalman filter (EKF) approach for SOC estimation.

\begin{tabular}{c} 
Initialization \\
1. Initialize with: \\
$\hat{x}_{0 \mid 0}=E\left(x_{0}\right), P_{0 \mid 0}=E\left[\left(x_{0}-\hat{x}_{0 \mid 0}\right)\left(x_{0}-\hat{x}_{0 \mid 0}\right)^{T}\right]$ \\
\hline State prediction \\
\hline 1. Update the step state vector: \\
$\hat{x}_{k \mid k-1}=A_{k-1} \hat{x}_{k-1 \mid k-1}+B_{k-1} u_{k-1}$ \\
2. Update the step error covariance: \\
$P_{k \mid k-1}=A_{k-1} P_{k-1 \mid k-1} A_{k-1}^{T}+\Gamma_{k-1} Q_{k-1} \Gamma_{k-1}^{T}$ \\
Measurement update \\
1. Calculate the Kalman gain: \\
$K_{k}=P_{k \mid k-1} C_{k}^{T}\left(C_{k} P_{k \mid k-1} C_{k}^{T}+R_{k}\right)$ \\
2. Update the measurement vector: \\
$\hat{x}_{k \mid k}=\hat{x}_{k \mid k-1}+K_{k}\left(y_{k}-g\left(x_{k}, u_{k}\right)\right)$ \\
3. Update the error covariance: \\
$P_{k \mid k}=\left(1-K_{k} C_{k}\right) P_{k \mid k-1}$ \\
\hline
\end{tabular}

The key point for implementing the EKF to our proposed model is to deal with the $C_{k}$. The detailed derivation process is below:

Assume that the vector of the parameters is $\theta=\left[R_{0}^{+}, R_{0}^{-}, M, \lambda\right]$, where $R_{0}^{+}$is the ohmic resistance when the cell is charging and $R_{0}^{-}$is the ohmic resistance when the cell is discharging. Then, to calculate $C_{k}^{\theta}$ :

$$
\begin{gathered}
\frac{d g\left(x_{k}, u_{k}, \theta\right)}{d \theta}=\frac{\partial g\left(x_{k}, u_{k}, \theta\right)}{\partial \theta}+\frac{\partial g\left(x_{k}, u_{k}, \theta\right)}{\partial x_{k}} \frac{d x_{k}}{d \theta} \\
\frac{d x_{k}}{d \theta}=\frac{\partial f\left(x_{k-1}, u_{k-1}, \theta\right)}{\partial \theta}+\frac{\partial f\left(x_{k-1}, u_{k-1}, \theta\right)}{\partial x_{k-1}} \frac{d x_{k-1}}{d \theta}
\end{gathered}
$$

The equation can be calculated recursively, and the initial value of $\frac{d x_{k}}{d \theta}(k=0)$ can be set to zero. In this particular case:

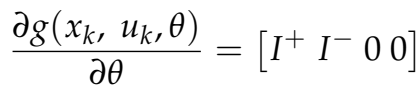

$$
\begin{aligned}
& \frac{\partial g\left(x_{k}, u_{k}, \theta\right)}{\partial x_{k}}=\left[11 \frac{\partial O C V\left(S_{k}\right)}{\partial S_{k}}\right] \\
& \frac{\partial f\left(x_{k-1}, u_{k-1}, \theta\right)}{\partial \theta} \\
& =\left[\begin{array}{cccc}
0 & 0 & 0 \\
0 & 0 & \left(1-\exp \left(\left|\frac{\eta i_{k-1} \gamma T_{s}}{C_{N}}\right|\right)\right) \operatorname{sgn}\left(i_{k-1}\right) & \left(h_{k-1}-M(S, \hat{S})\right) \\
0 & 0 & 0 & \left|\frac{\eta i_{k-1} T_{s}}{C_{N}}\right| \exp \left(\left|\frac{\eta i_{k-1} \gamma T_{s}}{C_{N}}\right|\right)
\end{array}\right] \\
& \frac{\partial f\left(x_{k-1}, u_{k-1}, \theta\right)}{\partial x_{k-1}}=\left[\begin{array}{ccc}
\exp \left(-\frac{T_{s}}{R_{1} C_{1}}\right) & 0 & 0 \\
0 & \exp \left(-\left|\frac{\eta i_{k} \gamma T_{s}}{C_{N}}\right|\right) & 0 \\
0 & 0 & 1
\end{array}\right]
\end{aligned}
$$

With the above equations, EKF can be executed recursively by repeating the steps in Table 2.

\subsection{Unscented Kalman Filtering}

Kalman filter is an optimum state recursive observer, and the frameworks of both EKF and UKF have a similar prediction-update structure. Alternatively, UKF linearized the system by unscented 
transform (UT) rather than the Taylor expansion adopted by EKF. UKF not only achieves a higher-order approximation expansion but also avoids the need to compute the Jacobian matrix. Based on the same discretized state-space Equations (13) and (14), the UKF algorithm steps are as follows:

Let $\chi$ be a n-dimension vector which obeys $X=\mathrm{N}(\chi, P)$ distribution.

Initialization:

$$
\bar{x}_{0}=E\left(x_{0}\right), P_{0}=\left[\left(x_{0}-\bar{x}_{0}\right)\left(x_{0}-\bar{x}_{0}\right)^{T}\right]
$$

Generate the sigma points via:

$$
\chi_{i, k-1}=\left\{\begin{array}{cl}
\bar{x}_{k-1}, & i=0 \\
\bar{x}_{k-1}+\left(\sqrt{(n+\lambda) P_{k-1}}\right), & i=1,2, \ldots, n \\
\bar{x}_{k-1}-\left(\sqrt{(n+\lambda) P_{k-1}}\right), & i=n+1, \ldots, 2 n
\end{array}\right.
$$

And their weights are computed via:

$$
\begin{gathered}
\left\{\begin{array}{c}
W_{0}^{m}=\frac{\lambda}{(n+\lambda)} \\
W_{i}^{m}=\frac{1}{2(n+\lambda)} \quad i=1, \ldots, 2 n
\end{array}\right. \\
\begin{cases}W_{0}^{c}=\frac{\lambda}{(n+\lambda)}+\left(1-\alpha^{2}+\beta\right) \\
W_{i}^{c}=\frac{1}{2(n+\lambda)} & i=1, \ldots, 2 n\end{cases}
\end{gathered}
$$

where $\lambda$ is the scaling parameter, which satisfies:

$$
\lambda=\alpha^{2}(n+\kappa)-n
$$

$\alpha$ is a small positive number $\left(10^{-4} \leq \alpha \leq 1\right)$. $\beta$ is used for absorbing a priori information for variable $\chi$. For Gaussian distribution, $\beta=2$ is optimal. $\kappa$ is a scaling factor that determine the degree of freedom of the sigma points, usually set 0 or $3-n$ to guarantee the positive definiteness of output variable covariance.

Prediction update:

$$
\begin{gathered}
\chi_{i, k \mid k-1}=f\left(x_{i, k-1}, u_{k}\right) \quad i=0, \ldots, 2 n \\
\bar{x}_{k \mid k-1}=\sum_{i=0}^{2 n} W_{i}^{m} \chi_{i, k \mid k-1}
\end{gathered}
$$

Update the error covariance:

$$
P_{k \mid k-1}=\sum_{i=0}^{2 n} W_{i}^{c}\left(\chi_{i, k \mid k-1}-\bar{x}_{k \mid k-1}\right)\left(\chi_{i, k \mid k-1}-\bar{x}_{k \mid k-1}\right)^{T}+Q_{k}
$$

where $Q_{k}$ is the covariance matrix of the state noise.

Measurement update:

$$
\begin{gathered}
y_{i, k \mid k-1}=g\left(x_{i, k-1}, u_{k}\right) \quad i=0, \ldots, 2 n \\
\bar{y}_{k \mid k-1}=\sum_{i=0}^{2 n} W_{i}^{m} y_{i, k-1}
\end{gathered}
$$

Calculate prediction covariance and cross-covariance:

$$
P_{y y, k}=\sum_{i=0}^{2 n} W_{i}^{c}\left(y_{i, k \mid k-1}-\bar{y}_{k \mid k-1}\right)\left(y_{i, k \mid k-1}-\bar{y}_{k \mid k-1}\right)^{T}+R_{k}
$$




$$
P_{x y, k}=\sum_{i=0}^{2 n} W_{i}^{c}\left(x_{i, k \mid k-1}-\bar{x}_{k \mid k-1}\right)\left(y_{i, k \mid k-1}-\bar{y}_{k \mid k-1}\right)^{T}
$$

where $R_{k}$ is the covariance matrix of the measurement noise.

Calculate the Kalman gain:

$$
K_{k}=\frac{P_{x y, k}}{P_{y y, k}}
$$

State estimate measurement update:

$$
\bar{x}_{k \mid k}=\bar{x}_{k \mid k-1}+K_{k}\left(y_{k}-\bar{y}_{k \mid k-1}\right)
$$

Error covariance measurement update:

$$
P_{k \mid k}=P_{k \mid k-1}-K_{k} P_{y y, k} K_{k}^{T}
$$

The UKF calculation procedure is summarized in Table 3.

Table 3. Summary of the unscented Kalman filter (UKF) approach for SOC estimation.

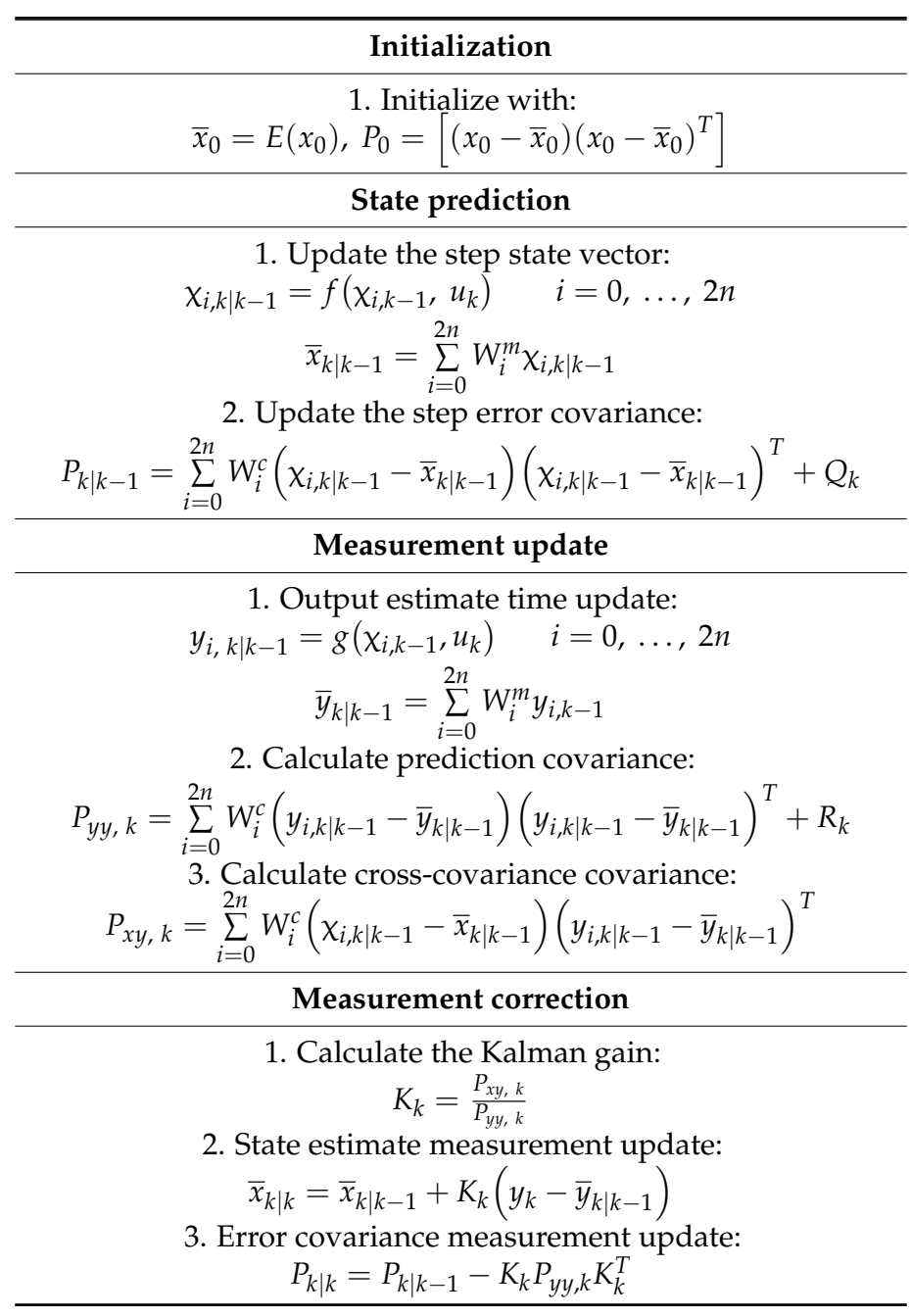

\subsection{Strong Tracking Unscented Kalman Filtering}

Although UKF can achieve better performance than EKF in estimation accuracy, it should be mentioned that UKF could lose the tracking capability and fail to converge the real value when it comes 
to the scenario that there is an abnormal change in one of the state vector components. Unfortunately, in real-world conditions, batteries suffer from highly dynamic current load that results in sudden acceleration and deceleration of driver intent. For the algorithm serve in a BMS, the sudden change of state vector, as well as the measurement error caused by the transducer, may lead to poor performance or biased results. Therefore, the STF is introduced in this study to address this problem. The idea of STF is to adjust Kalman gain matrix $K_{k}$ by introducing the suboptimal multiple fading factors into the covariance matrix $P_{k \mid k-1}$ [30]. With the adaptively modifying Kalman gain matrix and priori covariance matrix online, STF is able to resist the sudden change of system state vectors. The algorithm for $\mu_{k}$ and $P_{k \mid k-1}$ are as follows [30,32]:

Firstly, define the residual error $\varepsilon_{k}$ and residual error sequence covariance matrix $V_{k}$ :

$$
\begin{gathered}
\varepsilon_{k}=y_{k}-\bar{y}_{k \mid k-1} \\
V_{k}=\left\{\begin{array}{cc}
\varepsilon_{k} \varepsilon_{k}^{T} & k=1 \\
\frac{\rho V_{k-1}+\varepsilon_{k} \varepsilon_{k}^{T}}{1+\rho} & k \geq 2
\end{array}\right.
\end{gathered}
$$

Generally, set $\rho=0.95$.

Secondly, define the matrixes $N_{k}$ and $M_{k}$ as:

$$
\left\{\begin{array}{c}
N_{k}=V_{k}-C_{k} Q_{k-1} C_{k}^{T}-R_{k} \\
M_{k}=C_{k} P_{k \mid k-1} C_{k}^{T}+R_{k}-V_{k}+N_{k}
\end{array}\right.
$$

Lastly, the fading factor $\mu_{k}$ is calculated by the following equation:

$$
u_{k}=\left\{\begin{array}{cc}
\frac{\operatorname{tr}\left[N_{k}\right]}{\operatorname{tr}\left[M_{k}\right]} & u_{k} \geq 1 \\
1 & u_{k} \leq 1
\end{array}\right.
$$

where $\operatorname{tr}[\cdot]$ represents the trace of the matrix.

STF enforces the output residuals to be orthogonal or approximately orthogonal at each step to overcome the dynamic errors. The $P_{k \mid k-1}$ is recalculated by Equation (42) to replace Equation (30). The rest of the algorithm framework is the same as UKF algorithm.

$$
P_{k \mid k-1}=u_{k} \sum_{i=0}^{2 n} W_{i}^{c}\left(\chi_{i, k \mid k-1}-\bar{x}_{k \mid k-1}\right)\left(x_{i, k \mid k-1}-\bar{x}_{k \mid k-1}\right)^{T}+Q_{k}
$$

The process of STF for SOC online estimation is summarized in Figure 10. After the initialization with the state vector $\bar{x}_{0}$ and error covariance $P_{0}$, the data obtained by HPPC were used for model parameters update, then the fading factor was calculated according to the residual error $\varepsilon_{k}$. Afterward, the typical Kalman steps were performed while the a prior covariance matrix $P_{k \mid k-1}$ was corrected by fading factor. 


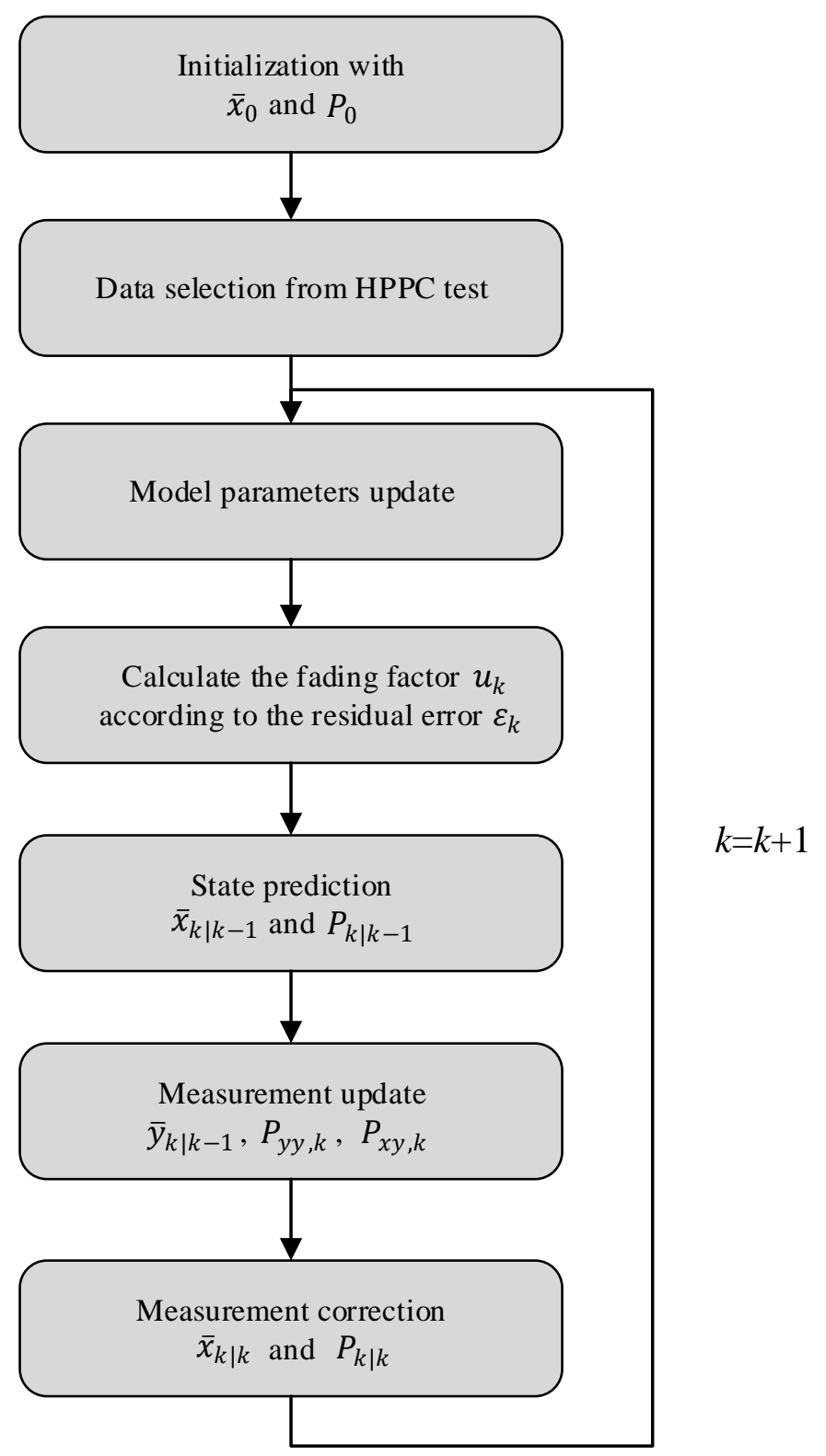

Figure 10. The flow chart of strong tracking filter (STF) algorithms.

\section{Results and Discussion}

The estimation performance of the three Kalman observers was compared. Since the true value of SOC in the validation test was calculated by the accumulative Ampere-hours flowing in and out of the battery, the test cells were fully charged by the CC-CV method and rested for $1 \mathrm{~h}$ at each test temperature to guarantee the initial $\mathrm{SOC}_{0}$ to be $100 \%$. The block diagram of our proposed SOC observers is illustrated in Figure 11. The validation test profile was loaded into the cells and Matlab/Simulink model, simultaneously. The sampling time was $1 \mathrm{~s}$, and the model parameters were updated according to the current SOC, I, and T. The parameters of each algorithm were configured as follows:

$$
Q_{k}=[0.10 ; 00.00001] ; R_{k}=0.001 ; \alpha=0.002 ; \beta=2 ; \kappa=1 .
$$




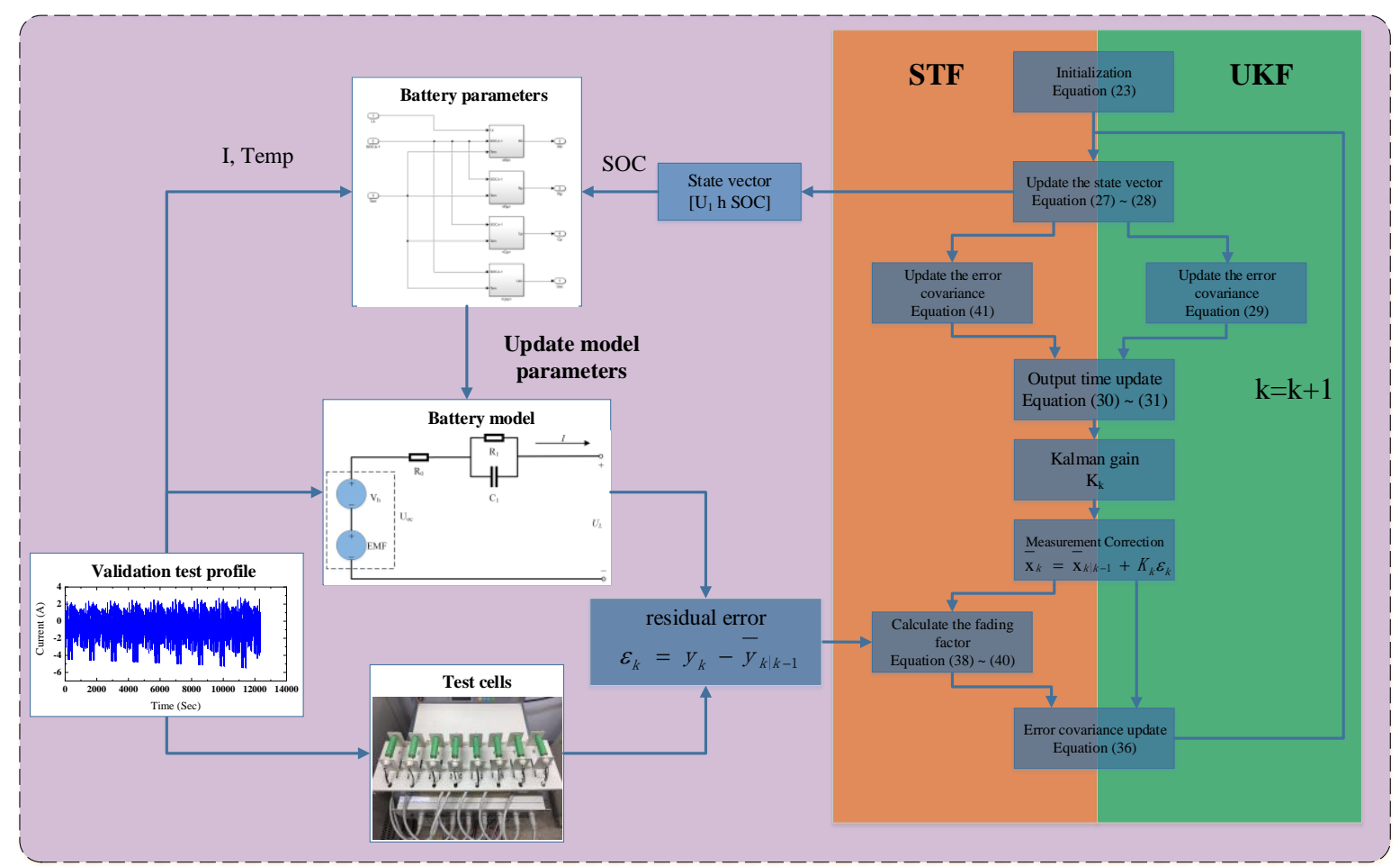

Figure 11. Block diagram of the proposed battery SOC observers.

The prediction accuracy of the three observers under each specific temperature was discussed. The comparative results between the experimental data obtained by the test equipment and the simulation data calculated by the three aforementioned algorithms under $0^{\circ} \mathrm{C}$ are shown in Figure 12a. Figure $12 \mathrm{~b}$ is the zoomed figure for Figure $12 \mathrm{a}$ from $4000 \mathrm{~s}$ to $6500 \mathrm{~s}$. As a result, it can be seen that the performance of STF was better than UKF, and UKF was better than EKF. As shown in Figure 5a, both charging and discharging processes existed in the FUDS cycles. However, the voltage presented a general descending trend which was corroborated by the SOC result as shown in Figure 12b. Hence, it can be said that EKF has the weakest capacity against the undulation which was caused by the charging and discharging conversion. The weakness in voltage tracking makes EKF curve the most fluctuant one in Figure 12b. Meanwhile, the estimation errors were also demonstrated in Figure 12c, and EKF has the lowest robustness to SOC fluctuation. The max data jitter reached $8 \%$ when the battery is working at low temperature. In addition, the MAE and RMSE were employed here again to evaluate the accuracy of EKF, UKF, and STF. Figure 13 shows the MAE and RMSE of the three algorithms at each temperature. For EKF and UKF, the MAE and RMSE decreased as the temperature increased. However, there was an anomaly that occurs in the case when the STF was at $0{ }^{\circ} \mathrm{C}$. We believe that the reason for the anomaly was that when cells were loading at $0^{\circ} \mathrm{C}$, the external performance deteriorated and the voltage fluctuation became more severe when the current direction changed. Consequently, the strong tracking factor $\mu_{k}$ began to step in and corrected the estimation results by adjusting the a prior covariance $P_{k \mid k-1}$. The statistical data of the MAE and RMSE are summarized in Table 4 . 
(a)
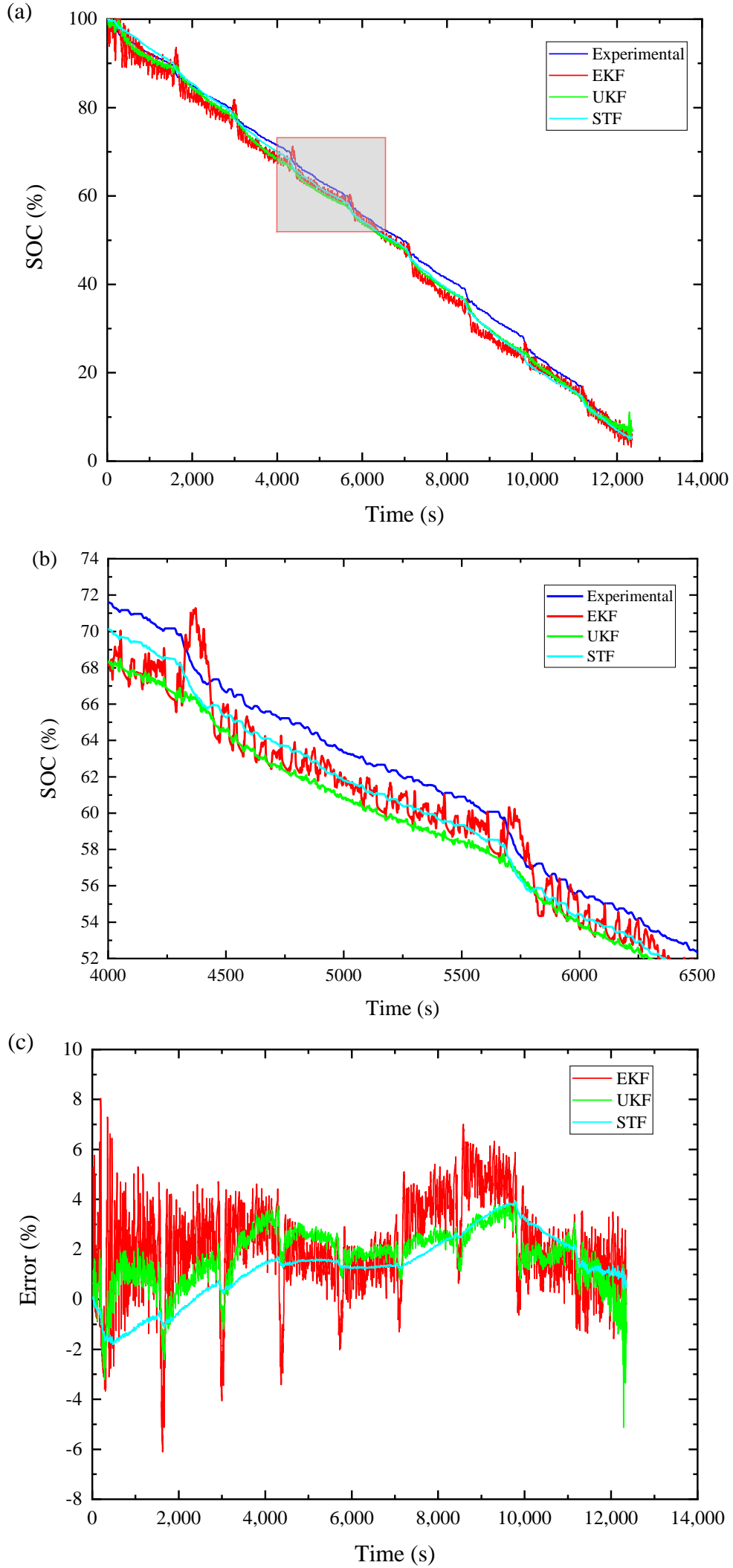

Figure 12. Comparative results of EKF, UKF, and STF for SOC estimation: (a) SOC estimation results; (b) the enlarged window from 4000 to $6500 \mathrm{~s}$; (c) SOC estimation error. 


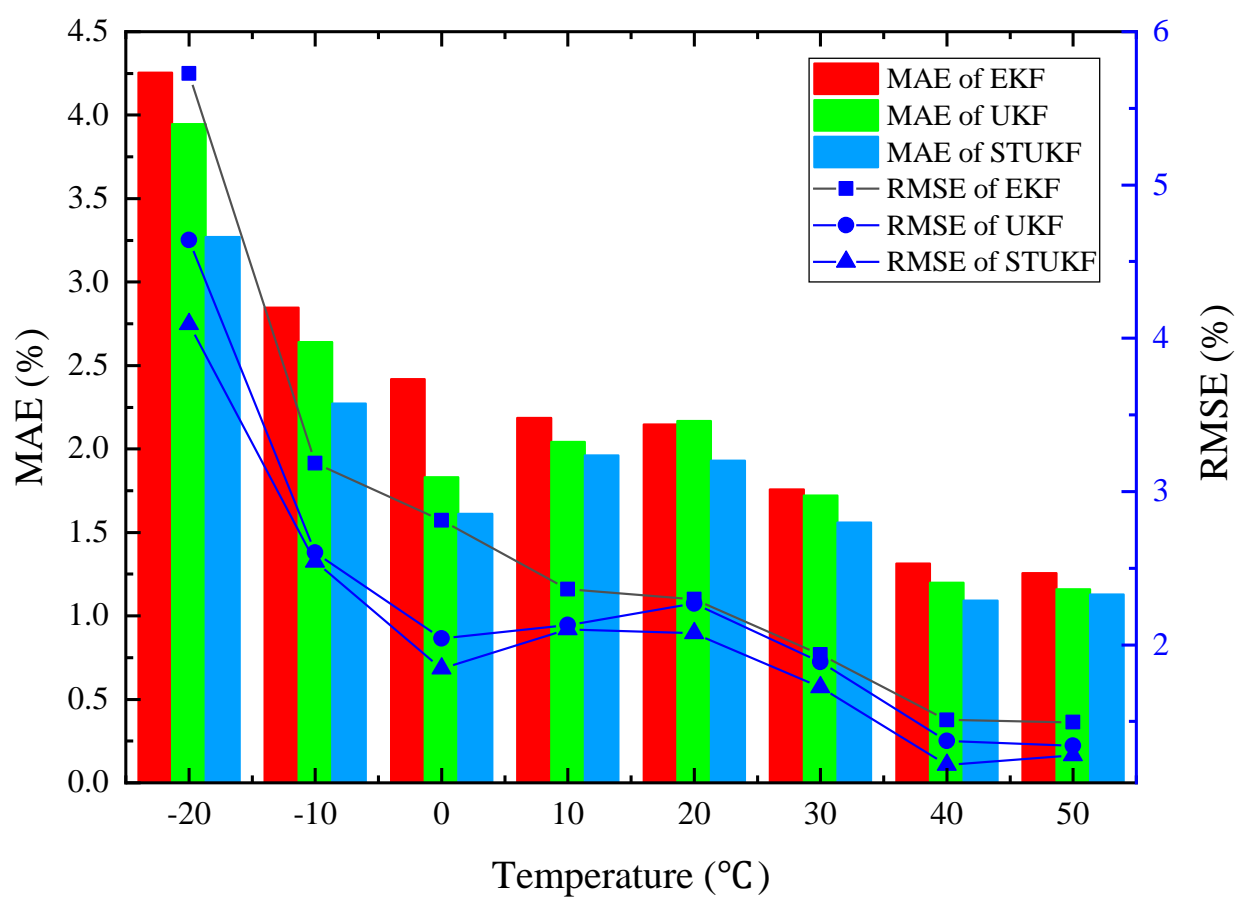

Figure 13. The MAE and RMSE of EKF, UKF, and STF at different temperatures.

Table 4. The statistical data of MAE and RMSE for EKF, UKF, and STF.

\begin{tabular}{|c|c|c|c|c|c|c|c|c|}
\hline \multicolumn{9}{|c|}{ MAE } \\
\hline & $-20^{\circ} \mathrm{C}$ & $-10{ }^{\circ} \mathrm{C}$ & $0^{\circ} \mathrm{C}$ & $10^{\circ} \mathrm{C}$ & $20^{\circ} \mathrm{C}$ & $30^{\circ} \mathrm{C}$ & $40^{\circ} \mathrm{C}$ & $50^{\circ} \mathrm{C}$ \\
\hline EKF & 4.2556 & 2.8469 & 2.4179 & 2.1860 & 2.1458 & 1.7587 & 1.3136 & 1.2558 \\
\hline UKF & 3.9459 & 2.6408 & 1.8307 & 2.0419 & 2.1688 & 1.7202 & 1.1985 & 1.1593 \\
\hline STF & 3.2692 & 2.2711 & 1.6102 & 1.9618 & 1.9292 & 1.5580 & 1.0925 & 1.1274 \\
\hline \multicolumn{9}{|c|}{ RMSE } \\
\hline & $-20^{\circ} \mathrm{C}$ & $-10{ }^{\circ} \mathrm{C}$ & $0^{\circ} \mathrm{C}$ & $10^{\circ} \mathrm{C}$ & $20^{\circ} \mathrm{C}$ & $30^{\circ} \mathrm{C}$ & $40^{\circ} \mathrm{C}$ & $50^{\circ} \mathrm{C}$ \\
\hline EKF & 5.7263 & 3.1847 & 2.8112 & 2.3636 & 2.2978 & 1.9360 & 1.5081 & 1.4964 \\
\hline UKF & 4.6393 & 2.6023 & 2.0419 & 2.1297 & 2.2687 & 1.8886 & 1.3736 & 1.3426 \\
\hline STF & 4.0924 & 2.5410 & 1.8461 & 2.1017 & 2.0763 & 1.7240 & 1.2160 & 1.2795 \\
\hline
\end{tabular}

In real applications, it is impossible to obtain the true initial $\mathrm{SOC}_{0}$ before use. Therefore, the robustness against the unknown initial $\mathrm{SOC}_{0}$ was also a crucial indicator of the estimation algorithms. Taking the experimental data from $0{ }^{\circ} \mathrm{C}$ as examples, Figure 14 shows the comparative results of the three algorithms with a SOC guess of $80 \%$ at $0{ }^{\circ} \mathrm{C}$. From Figure $14 \mathrm{a}$, all three algorithms could trace the true trajectory accurately and converge to the true value quickly even with large initial errors. From the enlarged window of 0 to $1500 \mathrm{~s}$ as shown in Figure 14b, it was clear that STF and UKF converged faster than EKF. Figure 14c demonstrates the estimation errors; the RMSEs of STF and UKF were $1.98 \%$ and $2.26 \%$, respectively, which were $48.28 \%$ and $29.91 \%$ smaller than those of the EKF. The simulation results were consistent with the fact that UT transformation, which is utilized by UKF and STF, will produce less truncation error than first-order Taylor expansion, which is employed by EKF. 

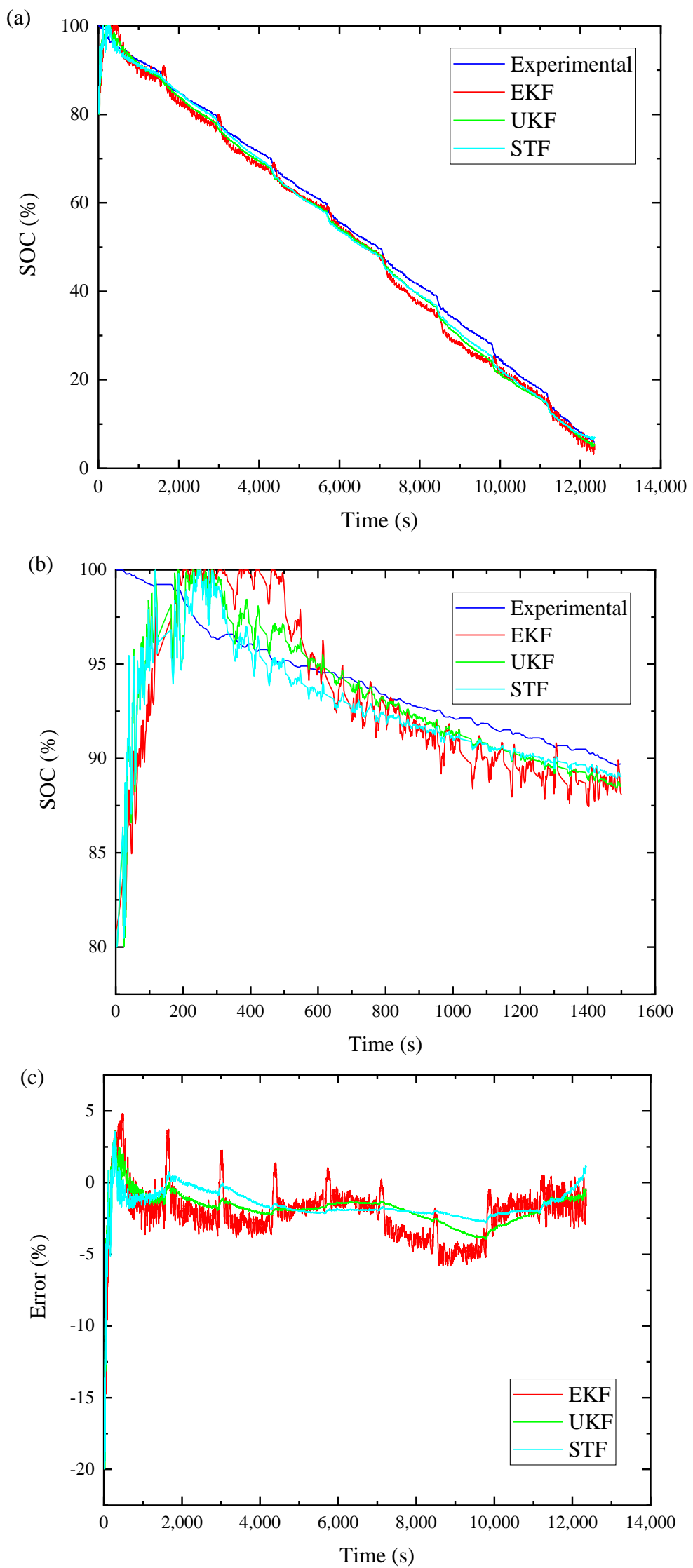

Figure 14. Robustness performance results with a SOC guess of $80 \%$ at a temperature of $0{ }^{\circ} \mathrm{C}$ : (a) SOC estimation results; (b) the enlarged window from 0 to $1500 \mathrm{~s}$; (c) SOC estimation error. 
To further discuss this topic, the initial SOC guess was set to $80 \%, 50 \%$, and $30 \%$, respectively. Figure 15 shows the part of the estimation results, and all the cases were summarized in Table 5 for comparison. There was an interesting point that could be noted in Figure 15. Regardless of the initial SOC guess values, all the curves converged at a specific point. After that point, the estimated curves present overlapped. We called this occurrence the convergence point, because it represented the convergence time of the algorithm. Judging from the position of the convergence point, the convergence times of UKF and STF were almost the same but a bit less than that of EKF. All the three algorithms were less than $2.5 \%$ of the whole operation time. This result is satisfactory for BMS applications.

(a)

EKF

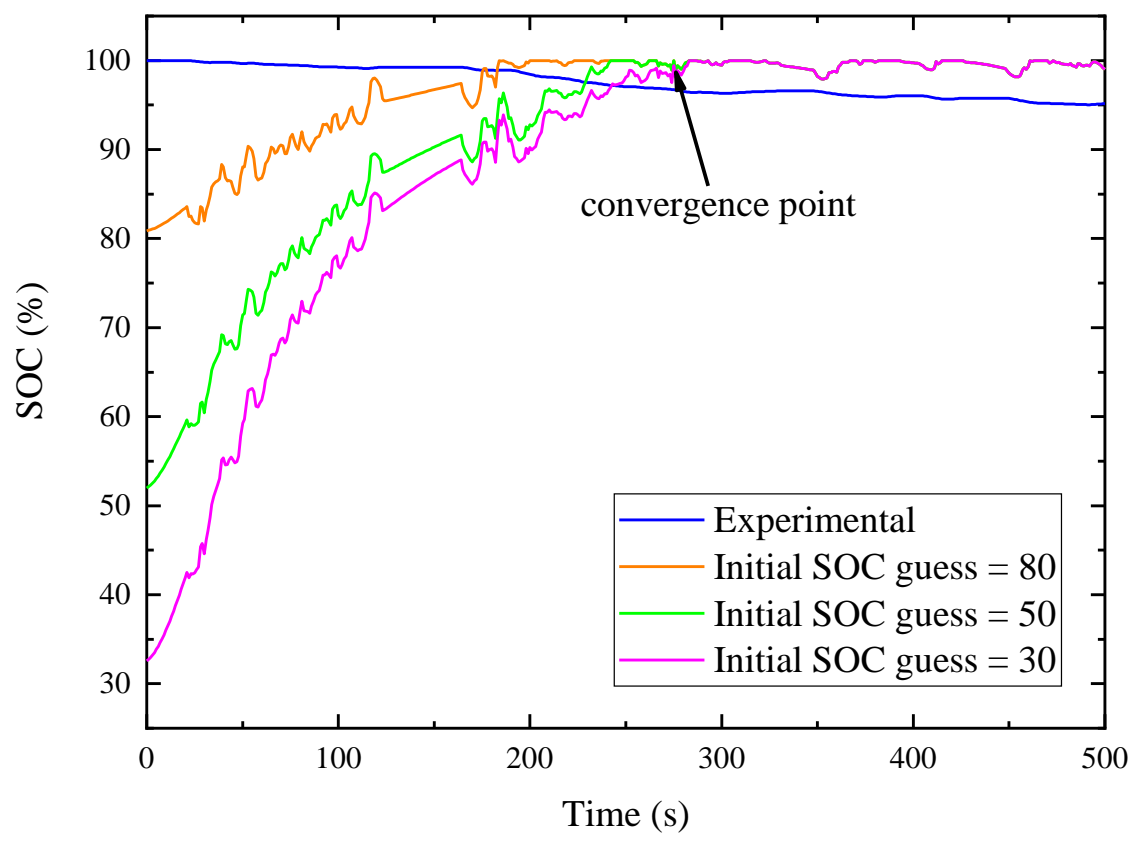

(b)

UKF

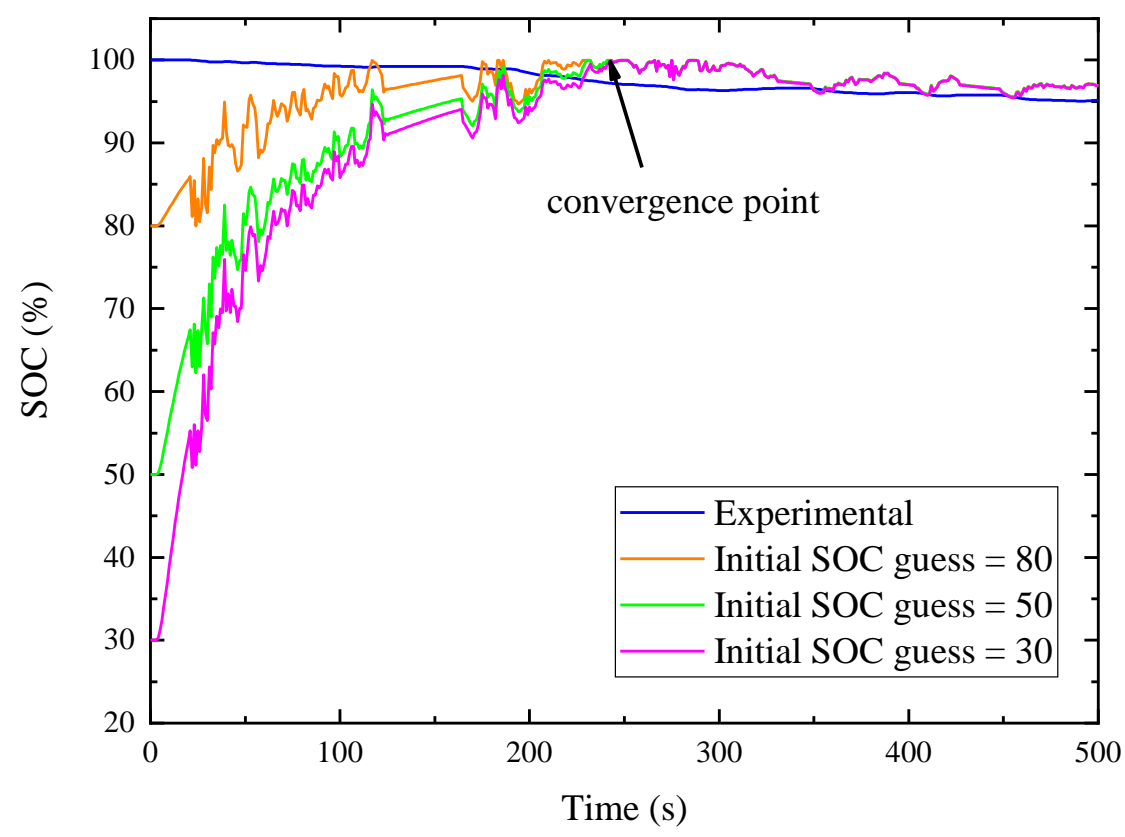

Figure 15. Cont. 
(c) STF

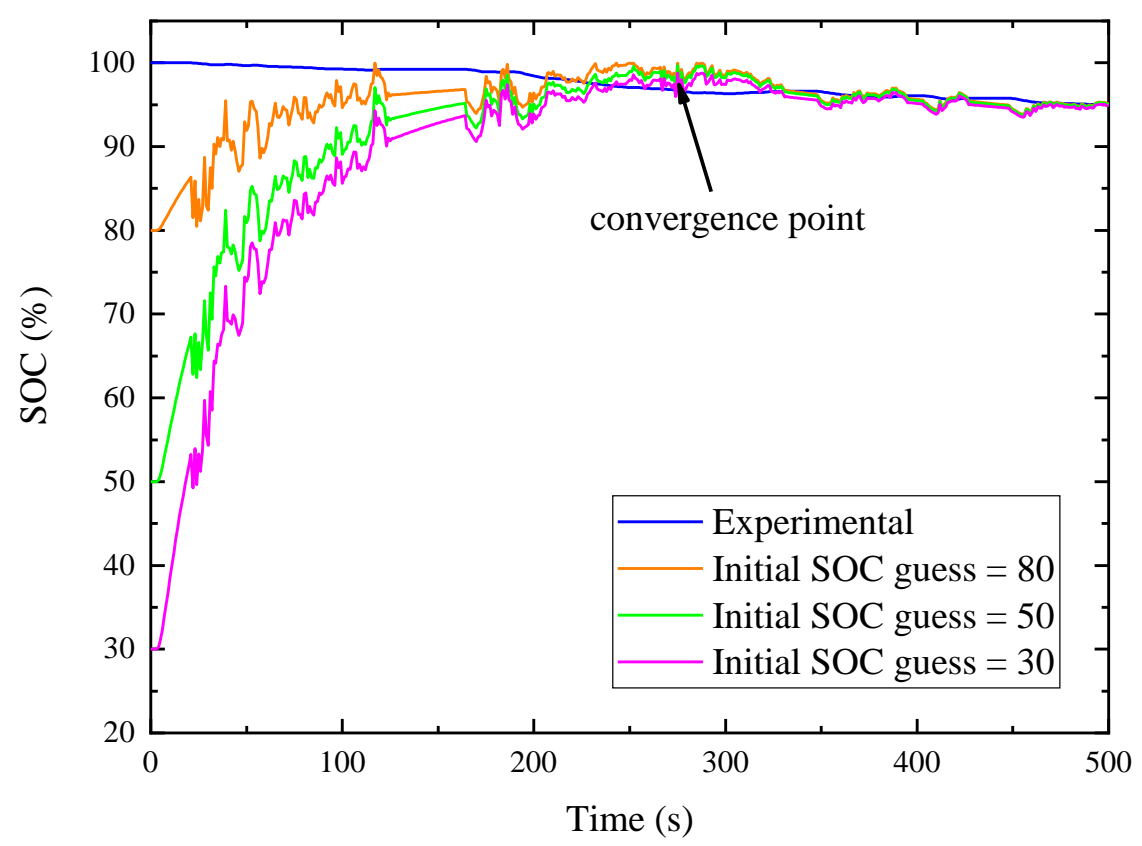

Figure 15. The robustness results for three algorithms: (a) EKF; (b) UKF; (c) STF.

Table 5. The MAE and RMSE by EKF, UKF, and STF at $0{ }^{\circ} \mathrm{C}$.

\begin{tabular}{cccc}
\hline \multicolumn{4}{c}{ MAE } \\
\hline Initial SOC Guess (\%) & EKF & UKF & STF \\
80 & 2.431 & 1.906 & 1.603 \\
50 & 2.632 & 2.043 & 1.732 \\
30 & 2.761 & 2.122 & 1.826 \\
\hline \multicolumn{4}{c}{ RMSE } \\
\hline Initial SOC Guess (\%) & EKF & UKF & STF \\
80 & 2.936 & 2.260 & 1.980 \\
50 & 4.045 & 3.173 & 2.967 \\
30 & 5.032 & 3.884 & 3.805 \\
\hline
\end{tabular}

Finally, we were also interested in the robustness against the voltage measurement signal of the three algorithms. The quality of the acquired voltage signals was always a challenge for BMS, especially when the BMS was exposed to the vehicle's high electromagnetic interference environment. Hence, a constant $-5 \mathrm{mV}$ voltage measurement offset is designed to simulated the real-world voltage sensor drift in the BMS. We used the test datasheet of $30^{\circ} \mathrm{C}$ for validation. The voltage offset data was embedded in the validation test datasheet of $30{ }^{\circ} \mathrm{C}$ with the initial SOC at $100 \%$.

As shown in Figure 16, the STF had minimum error and the fastest convergence rate. Moreover, the MAE and RMSE of the three algorithms were tabulated in Table 6. With the same initial SOC, the MAE and RMSE of three algorithms were arranged from large to small by EKF, UKF, and STF sequence, thereby suggesting that STF was the most robust. 


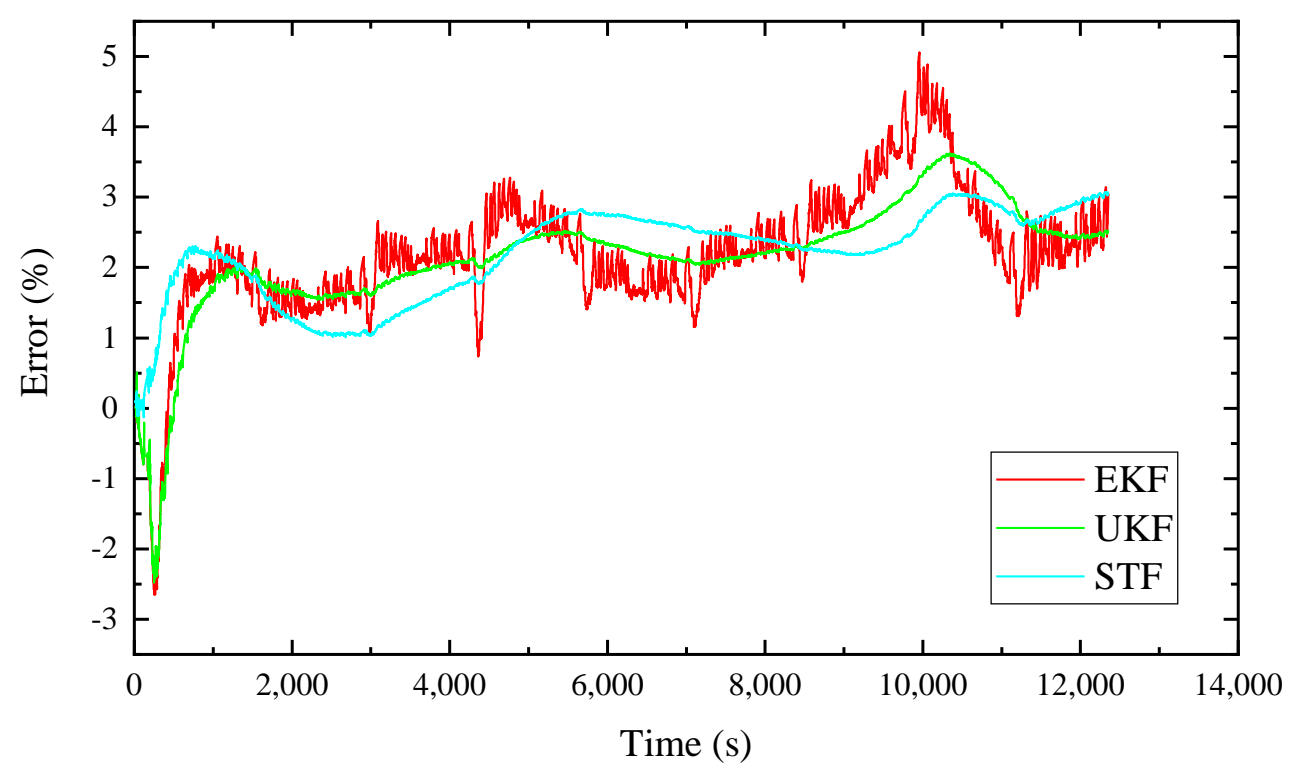

Figure 16. The estimated SOC error of the algorithms with $-5 \mathrm{mV}$ voltage offset at $30{ }^{\circ} \mathrm{C}$.

Table 6. The MAE and RMSE of three algorithms when with voltage measurement offset.

\begin{tabular}{ccc}
\hline Algorithm & MAE (\%) & RMSE (\%) \\
\hline EKF & 2.262 & 2.385 \\
UKF & 2.222 & 2.305 \\
STF & 2.186 & 2.280 \\
\hline
\end{tabular}

\section{Conclusions}

We presented a modified ECM that took hysteresis and temperature effect into account for the first time. The proposed model aimed to improve the voltage tracking accuracy of the existing ECM whose applicability is often limited to steady current profiles and certain temperatures. With the offline hysteresis-SOC-T table correcting the OCV and the rest of the model parameters were estimated online, the improved model has the adaptability to ambient temperature rather than origin model. Then the improved model was applied to three model-based recursive estimators including EKF, UKF, and STF for SOC estimation. The procedures to execute each step for parameter identification and the algorithms were explained in detail. Lastly, the model and the estimators were validated by experimental test with commercial 18650 type $\mathrm{Li}\left(\mathrm{Ni}_{1 / 3} \mathrm{Co}_{1 / 3} \mathrm{Mn}_{1 / 3}\right) \mathrm{O}_{2}$ cells. The validation test consisted of several high dynamic FUDS microcycles, rest period, and a discharge process. This test was carried out under different temperatures ranging from $0{ }^{\circ} \mathrm{C}$ to $50{ }^{\circ} \mathrm{C}$. The MAE and RMSE of the proposed model were smaller than the ordinary model in voltage estimation. In the same vein, the performances of EKF, UKF, and STF were also evaluated by MAE and RMSE. The STF algorithm outperformed UKF and EKF in estimation accuracy, robustness, and convergence behavior, thereby demonstrating a better solution for BMS application.

Author Contributions: C.Z. designed the experiments and wrote the paper; F.Y. and C.D. dominated and supervised the project; G.R. help to review and edit the paper.

Funding: This work was funded by the National Natural Science Foundation of China, grant number 51775393; the platform construction of Hubei province renewable energy intelligent vehicle, grant number 2016BEC116; the 111 Project, grant number B17034 and Research and Development of Products Test Cycle for New Energy Vehicles in China and the Fundamental Research Funds for the Central Universities, grant number WUT:2017-IVA-034.

Conflicts of Interest: The authors declare no conflicts of interest. 


\section{Nomenclature}

$\mathrm{C}_{\mathrm{N}} \quad$ Battery nominal capacity

$U_{L} \quad$ terminal voltage

$U_{1} \quad$ voltage over RC network

$U_{o c} \quad$ open circle voltage

$R_{1} \quad$ polarization resistance

$C_{1} \quad$ polarization capacitance

$\mathrm{I}^{+} \quad$ battery charge current

$I^{-} \quad$ battery discharge current

$k$ time step index

$\eta \quad$ coulomb efficiency

$h_{\mathrm{k}} \quad$ hysteresis voltage

$\gamma \quad$ positive constant to tune the rate of decay

$S(k) \quad$ experimental data (battery voltage or SOC) at step $k$

$\hat{S}(k) \quad$ estimated value (battery voltage or SOC) at step $k$

$k \mid k-1 \quad$ prior estimation state

$\omega_{k} \quad$ system process noise

$v_{k} \quad$ measurement noise

$\xi \quad$ identification parameters

$\chi \quad$ a n-dimension vector for state space

$\lambda \quad$ scaling parameter

$W_{i}^{m} \quad$ weights of mean

$W_{i}^{c} \quad$ weights of covariance

$\alpha \quad$ small positive number

$\beta \quad$ state distribution parameter

$\kappa \quad$ scaling factor

$Q_{k} \quad$ covariance matrix of the state noise

$R_{k} \quad$ covariance matrix of the measurement noise

$\mathrm{K}_{k} \quad$ Kalman gain

$\varepsilon_{\mathrm{k}} \quad$ residual error

$V_{\mathrm{k}} \quad$ residual error sequence covariance matrix

$\mu_{k} \quad$ fading factor

List of abbreviations

ANN Artificial Neural Network

BMS Battery Management System

BSF Battery Size Factor

CDKF Central-Difference Kalman filter

ECM Equivalent Circuit Model

EKF Extended Kalman Filter

EMF Electro-Motive Force

EV Electric Vehicles

FL Fuzzy Logic

FUDS Federal Urban Driving Schedule

HPPC Hybrid Pulse Power Characterization

KF Kalman filter

MAE Mean Absolute Error

OCV Open Circuit Voltage

RLS Least Square Method

RMSE Root Mean Squared Error

SOC State of Charge

STF Strong Tracking Filter

SVM Support Vector Machine

UKF Unscented Klaman Filter 


\section{References}

1. Li, Z.; Huang, J.; Liaw, B.Y.; Zhang, J. On state-of-charge determination for lithium-ion batteries. J. Power Sources 2017, 348, 281-301. [CrossRef]

2. Waag, W.; Fleischer, C.; Sauer, D.U. Critical review of the methods for monitoring of lithium-ion batteries in electric and hybrid vehicles. J. Power Sources 2014, 258, 321-339. [CrossRef]

3. Ismail, M.M.; Hassan, M.A.M. The state of charge estimation for rechargeable batteries based on artificial neural network techniques. In Proceedings of the IEEE Control Decision and Information Technologies (CoDIT), Hammamet, Tunisia, 6-8 May 2013; pp. 733-739.

4. Ismail, M.; Dlyma, R.; Elrakaybi, A. Battery state of charge estimation using an Artificial Neural Network. In Proceedings of the 2017 IEEE Transportation Electrification Conference and Expo (ITEC), Chicago, IL, USA, 22-24 June 2017.

5. Burgos, C.; Sáez, D.; Orchard, M.E.; Cárdenas, R. Fuzzy modelling for the state-of-charge estimation of lead-acid batteries. J. Power Sources 2015, 274, 355-366. [CrossRef]

6. Jiani, D.; Zhitao, L.; Youyi, W. A fuzzy logic-based model for Li-ion battery with SOC and temperature effect. In Proceedings of the 11th IEEE Control \& Automation (ICCA), Taichung, Taiwan, 18-20 June 2014; pp. 1333-1338.

7. Álvarez Antón, J.C.; García Nieto, P.J.; de Cos Juez, F.J.; Sánchez Lasheras, F.; González Vega, M.; Roqueñí Gutiérrez, M.N. Battery state-of-charge estimator using the SVM technique. Appl. Math. Modell. 2013, 37, 6244-6253. [CrossRef]

8. Hansen, T.; Wang, C.-J. Support vector based battery state of charge estimator. J. Power Sources 2005, 141, 351-358. [CrossRef]

9. Sheng, H.; Xiao, J. Electric vehicle state of charge estimation: Nonlinear correlation and fuzzy support vector machine. J. Power Sources 2015, 281, 131-137. [CrossRef]

10. Surendar, V.; Mohankumar, V.; Anand, S.; Prasanna, V.D. Estimation of State of Charge of a Lead Acid Battery Using Support Vector Regression. Procedia Technol. 2015, 21, 264-270. [CrossRef]

11. Alvarez Anton, J.C.; Garcia Nieto, P.J.; Blanco Viejo, C.; Vilan Vilan, J.A. Support Vector Machines Used to Estimate the Battery State of Charge. IEEE Trans. Power Electron. 2013, 28, 5919-5926. [CrossRef]

12. Campestrini, C.; Horsche, M.F.; Zilberman, I.; Heil, T.; Zimmermann, T.; Jossen, A. Validation and benchmark methods for battery management system functionalities: State of charge estimation algorithms. J. Energy Storage 2016, 7, 38-51. [CrossRef]

13. Plett, G.L. Extended Kalman filtering for battery management systems of LiPB-based HEV battery packs Part 1. Background. J. Power Sources 2004, 134, 252-261. [CrossRef]

14. Plett, G.L. Extended Kalman filtering for battery management systems of LiPB-based HEV battery packs Part 2. Modeling and identification. J. Power Sources 2004, 134, 262-276. [CrossRef]

15. Plett, G.L. Extended Kalman filtering for battery management systems of LiPB-based HEV battery packs Part 3. State and parameter estimation. J. Power Sources 2004, 134, 277-292. [CrossRef]

16. Plett, G.L. High-Performance Battery-Pack Power Estimation Using a Dynamic Cell Model. IEEE Trans. Veh. Technol. 2004, 53, 1586-1593. [CrossRef]

17. Dai, H.; Sun, Z.; Wei, X. Online SOC Estimation of High-power Lithium-ion Batteries Used on HEVs. IEEE Veh. Electron. Saf. 2006, 342-347.

18. Mastali, M.; Vazquez-Arenas, J.; Fraser, R.; Fowler, M.; Afshar, S.; Stevens, M. Battery state of the charge estimation using Kalman filtering. J. Power Sources 2013, 239, 294-307. [CrossRef]

19. Sepasi, S.; Ghorbani, R.; Liaw, B.Y. Improved extended Kalman filter for state of charge estimation of battery pack. J. Power Sources 2014, 255, 368-376. [CrossRef]

20. Pérez, G.; Garmendia, M.; Reynaud, J.F.; Crego, J.; Viscarret, U. Enhanced closed loop State of Charge estimator for lithium-ion batteries based on Extended Kalman Filter. Appl. Energy 2015, 155, 834-845. [CrossRef]

21. Pan, H.; Lü, Z.; Lin, W.; Li, J.; Chen, L. State of charge estimation of lithium-ion batteries using a grey extended Kalman filter and a novel open-circuit voltage model. Energy 2017, 138, 764-775. [CrossRef]

22. Li, Z.; Zhang, P.; Wang, Z.; Song, Q.; Rong, Y. State of Charge Estimation for Li-ion Battery Based on Extended Kalman Filter. Energy Procedia 2017, 105, 3515-3520. 
23. Sun, F.; Hu, X.; Zou, Y.; Li, S. Adaptive unscented Kalman filtering for state of charge estimation of a lithium-ion battery for electric vehicles. Energy 2011, 36, 3531-3540. [CrossRef]

24. Xiong, R.; Gong, X.; Mi, C.C.; Sun, F. A robust state-of-charge estimator for multiple types of lithium-ion batteries using adaptive extended Kalman filter. J. Power Sources 2013, 243, 805-816. [CrossRef]

25. Roscher, M.A.; Sauer, D.U. Dynamic electric behavior and open-circuit-voltage modeling of LiFePO4-based lithium ion secondary batteries. J. Power Sources 2011, 196, 331-336. [CrossRef]

26. García-Plaza, M.; Eloy-García Carrasco, J.; Peña-Asensio, A.; Alonso-Martínez, J.; Arnaltes Gómez, S. Hysteresis effect influence on electrochemical battery modeling. Electr. Power Syst. Res. 2017, 152, 27-35. [CrossRef]

27. Dong, G.; Wei, J.; Zhang, C.; Chen, Z. Online state of charge estimation and open circuit voltage hysteresis modeling of LiFePO 4 battery using invariant imbedding method. Appl. Energy 2016, 162, 163-171. [CrossRef]

28. Li, H.; Song, Y.; Lu, B.; Zhang, J. Effects of stress dependent electrochemical reaction on voltage hysteresis of lithium ion batteries. Appl. Math. Mech. 2018, 39, 1453-1464. [CrossRef]

29. Christopherson, J.P. Battery Test Manual For Electric Vehicles; Idaho National Laboratory: Idaho Falls, ID, USA, 2015.

30. Li, Y.; Wang, C.; Gong, J. A combination Kalman filter approach for State of Charge estimation of lithium-ion battery considering model uncertainty. Energy 2016, 109, 933-946. [CrossRef]

31. Xiong, R.; Sun, F.; Gong, X.; Gao, C. A data-driven based adaptive state of charge estimator of lithium-ion polymer battery used in electric vehicles. Appl. Energy 2014, 113, 1421-1433. [CrossRef]

32. Li, D.; Ouyang, J.; Li, H.; Wan, J. State of charge estimation for LiMn2O4 power battery based on strong tracking sigma point Kalman filter. J. Power Sources 2015, 279, 439-449. [CrossRef]

(C) 2018 by the authors. Licensee MDPI, Basel, Switzerland. This article is an open access article distributed under the terms and conditions of the Creative Commons Attribution (CC BY) license (http:// creativecommons.org/licenses/by/4.0/). 